\title{
Condensate of massive graviton and dark matter
}

\author{
Katsuki Aoki ${ }^{*}$ and Kei-ichi Maeda \\ Department of Physics, Waseda University, Shinjuku, Tokyo 169-8555, Japan
}

(Received 24 July 2017; published 1 February 2018)

\begin{abstract}
We study coherently oscillating massive gravitons in the ghost-free bigravity theory. This coherent field can be interpreted as a condensate of the massive gravitons. We first define the effective energy-momentum tensor of the coherent massive gravitons in a curved spacetime. We then study the background dynamics of the Universe and the cosmic structure formation including the effects of the coherent massive gravitons. We find that the condensate of the massive graviton behaves as a dark matter component of the Universe. From the geometrical point of view the condensate is regarded as a spacetime anisotropy. Hence, in our scenario, dark matter is originated from the tiny deformation of the spacetime. We also discuss a production of the spacetime anisotropy and find that the extragalactic magnetic field of a primordial origin can yield a sufficient amount for dark matter.
\end{abstract}

DOI: 10.1103/PhysRevD.97.044002

\section{INTRODUCTION}

The existence of gravitational waves was indeed confirmed by the direct detections [1,2], and their quantum counterpart is called gravitons. The gravitons are defined by perturbations around a background spacetime. The effective energy-momentum tensor of the high-frequency gravitons in general relativity (GR) was derived by Isaacson $[3,4]$ which enables us to treat the gravitons as massless spin-2 particles whose energy and momentum change the background geometry. Due to the nonlinear features of the Einstein equations the effective energymomentum tensor cannot be straightforwardly defined. The gravitons are well defined when their frequencies (and their momenta) are high enough compared with the curvature scale of the background and then the energymomentum tensor is defined via a non-local operation which projects the nonlinear quantities of the gravitons onto those in low-frequency modes. However, the low energy states of gravitons, i.e., low frequency/momentum modes of gravitons, should be ill defined in GR. This is not the case when a graviton is massive.

Although GR is now widely accepted as a low-energy effective theory of gravity, the question whether the graviton is indeed massless or not has been long discussed (see [5-7] for reviews and [8-10] for experimental constraints on the

\footnotetext{
*katsuki-a12@gravity.phys.waseda.ac.jp maeda@waseda.ac.jp
}

Published by the American Physical Society under the terms of the Creative Commons Attribution 4.0 International license. Further distribution of this work must maintain attribution to the author(s) and the published article's title, journal citation, and DOI. Funded by SCOAP ${ }^{3}$. graviton mass). The linear theory of the massive spin-2 field was constructed by Fierz and Pauli in 1939 [11]. Since the gravity must be represented by a nonlinear theory of the metric tensor, the Fierz-Pauli theory requires an extension to the nonlinear theory of the metric in order to obtain the theory of the massive graviton. Generic nonlinear extension of the Fierz-Pauli theory turns to be unstable, called the BoulwareDeser ghost [12]. However, the ghost-free nonlinear massive gravity was proposed by de Rham et al. in $2010[13,14]$ which was further extended into the bigravity theory [15] and the multigravity theory [16]. In the bigravity theory or the multigravity theory, the gravity is still a long-range force because there exists a massless graviton in addition to the massive graviton(s). In the present paper, we focus on the bigravity theory which contains a massless graviton and a massive graviton. The effective energy-momentum tensors of both massless and massive gravitons are defined in the similar way to the case of GR [17].

The bigravity theory has received much attentions related to the discovery of dark energy and dark matter. If the graviton mass is extremely small such as $m \sim 10^{-33} \mathrm{eV}$, the present accelerating expansion of the Universe can be explained by the tiny graviton mass [18-25]. Other range of the mass may explain the origin of dark matter. For instance, dark matter is originated from a matter field in the "dark sector" when $m \gtrsim 10^{-27} \mathrm{eV}[25,26]$ whereas the massive graviton itself is a candidate of dark matter when $10^{-4} \mathrm{eV} \lesssim m \lesssim 10^{7} \mathrm{eV}$ [17] (see also [27,28]).

The first suggestion to dark matter in the ghost-free bigravity theory was given by [22] which found that the anisotropy of the spacetime behaves like a dust fluid as for the contribution to the Friedmann equation. However, the following questions have not been cleared: Why does the anisotropy behave as a nonrelativistic fluid? Whether or not 
can it explain other phenomena of dark matter, e.g., the cosmic structure formation? In the present paper, thus, we explore those questions and find that the dark matter component can be regarded as the "condensate" of the massive graviton and it can give local structures of the Universe.

We shall focus on the case when the massive graviton is dominated by the zero momentum mode; that is, the configuration of the massive graviton is almost homogeneous. This configuration can be interpreted as the condensate of the massive graviton which we call the massive graviton condensate. Contrary to the case of the massless graviton, the zero momentum mode of the massive graviton shows a coherent oscillation due to the mass term. Therefore, we can define the energy-momentum tensor of the zero momentum mode of the massive graviton as long as the graviton mass is larger than the curvature scale of the background spacetime. We find that the zero momentum mode of the massive graviton gives a dark matter contribution to the Fridemann equation and the tiny fluctuations around the zero momentum mode provide the cosmic structure formation. The constraint on the graviton mass to be dark matter is the same as that obtained in [17], i.e., $10^{-4} \mathrm{eV} \lesssim$ $m \lesssim 10^{7} \mathrm{eV}$, in general.

From the geometrical aspect the zero momentum mode of the massive graviton represents the anisotropy of the spacetime. The Universe filled with the zero momentum massive gravitons is interpreted as a homogeneous spacetime. The anisotropic component of the Universe acts as dark matter which is indeed shown by [22]. The metric perturbations around the homogeneous spacetime can provide the structures of the Universe.

The tiny anisotropy of the Universe can be produced when there is a coherent field with an anisotropic stress. A possible candidate of the source is the extragalactic magnetic field of a primordial origin (see e.g., [29-32]). Recent blazar observations implies the existence of the extragalactic magnetic field whose lower bound of the strength $B_{0}$ is about $10^{-17} \mathrm{G}$ [33-39]. This magnetic field could be produced in the early Universe [40,41]. We will show that the coherent magnetic field can yield a sufficient amount of the massive graviton condensate in order to explain the present abundance of dark matter.

The paper is organized as follows. After a brief introduction about the ghost-free bigravity theory in Sec. II, we define the effective energy-momentum tensor of the coherent massive graviton in Sec. III. The homogeneous configuration of $\varphi_{\mu \nu}$ is studied in Sec. IV which reproduces the result obtained by [22] from a field theoretical aspect. We then study the perturbations around the homogeneous mode in Sec. V. We show that the massive graviton condensate is indeed a viable candidate of dark matter. In Sec. VI, a production of the condensate to be dark matter is discussed. We give a summary and some discussions in Sec. VII. In Appendix A, we summarize the definitions of the energy-momentum tensors of the high-frequency massive and massless gravitons in a curved spacetime. We briefly study the Bianchi I universe in bigravity in Appendix B. In Appendix C, we detail the calculations about the inhomogeneous modes of the massive graviton condensate.

\section{BIGRAVITY THEORY}

The action of the bigravity theory proposed by Hassan and Rosen [15] is given by

$$
\begin{aligned}
S= & \frac{1}{2 \kappa_{g}^{2}} \int d^{4} x \sqrt{-g} R(g)+\frac{1}{2 \kappa_{f}^{2}} \int d^{4} x \sqrt{-f} \mathcal{R}(f) \\
& -\frac{m^{2}}{\kappa^{2}} \int d^{4} x \sqrt{-g} \mathcal{U}(g, f)+S^{[\mathrm{m}]},
\end{aligned}
$$

where $g_{\mu \nu}$ and $f_{\mu \nu}$ are two dynamical metrics, and $R(g)$ and $\mathcal{R}(f)$ are their Ricci scalars. The parameters $\kappa_{g}^{2}=8 \pi G$ and $\kappa_{f}^{2}=8 \pi \mathcal{G}$ are the corresponding gravitational constants, while $\kappa$ is defined by $\kappa^{2}=\kappa_{g}^{2}+\kappa_{f}^{2}$.

The ghost-free interaction term between the two metrics is given by

$$
\mathcal{U}(g, f)=\sum_{k=0}^{4} b_{k} \mathcal{U}_{k}\left(\sqrt{g^{-1} f}\right),
$$

where $\left\{b_{k}\right\}(k=0-4)$ are coupling constants and the $4 \times 4$ matrix $\sqrt{g^{-1} f}=\left(\sqrt{g^{-1} f}\right)^{\mu}{ }_{\nu}$ is defined by

$$
\left(\sqrt{g^{-1} f}\right)_{\rho}^{\mu}\left(\sqrt{g^{-1} f}\right)_{\nu}^{\rho}=g^{\mu \rho} f_{\rho \nu}
$$

while $\mathcal{U}_{k}$ are the elementary symmetric polynomials of the eigenvalues of the matrix $\sqrt{g^{-1} f}$.

Just for simplicity, we assume that matter is coupled only to the $g$-metric

$$
S^{[\mathrm{m}]}=S_{g}^{[\mathrm{m}]}\left(g, \psi_{g}\right) .
$$

We shall briefly discuss the case when other types of matter fields are introduced in Sec. VII and Appendix A. Our conclusion is not changed even for those cases.

The fully nonlinear equations of motion are given by

$$
\begin{gathered}
G^{\mu \nu}(g)=\kappa_{g}^{2}\left(T_{(\text {int) }}^{\mu \nu}+T^{\mu \nu}\right), \\
\mathcal{G}^{\mu \nu}(f)=\kappa_{f}^{2} \mathcal{T}_{\text {(int) }}^{\mu \nu},
\end{gathered}
$$

where $T^{\mu \nu}$ is the matter energy-momentum tensor while $T_{\text {(int) }}^{\mu \nu}$ and $\mathcal{T}_{\text {(int) }}^{\mu \nu}$ are derived by the variations of the interaction term $\mathcal{U}$ with respect to $g_{\mu \nu}$ and $f_{\mu \nu}$, respectively. The contracted Bianchi identity and the matter conservation law $\nabla_{\mu}^{(g)} T^{\mu \nu}=0$ lead to 


$$
\stackrel{(g)}{\nabla_{\mu}} T^{(\mathrm{int}) \mu \nu}=0, \quad \stackrel{(f)}{\nabla_{\mu}} \mathcal{T}^{(\mathrm{int}) \mu \nu}=0,
$$

where $\nabla_{\mu}$ and $\nabla_{\mu}$ are the covariant derivatives with respect to $g_{\mu \nu}$ and $f_{\mu \nu}$, respectively.

There is a particular vacuum solution in which two spacetimes are homothetic such that

$$
f_{\mu \nu}=\xi_{0}^{2} g_{\mu \nu},
$$

where $\xi_{0}$ is a root of the quartic equation

$$
\Lambda_{g}=\xi_{0}^{2} \Lambda_{f}
$$

with

$$
\begin{gathered}
\Lambda_{g}:=m^{2} \frac{\kappa_{g}^{2}}{\kappa^{2}}\left(b_{0}+3 b_{1} \xi_{0}+3 b_{2} \xi_{0}^{2}+b_{3} \xi_{0}^{3}\right), \\
\Lambda_{f}:=m^{2} \frac{\kappa_{f}^{2}}{\kappa^{2}}\left(b_{4}+3 b_{3} \xi_{0}^{-1}+3 b_{2} \xi_{0}^{-2}+b_{1} \xi_{0}^{-3}\right) .
\end{gathered}
$$

For the homothetic solutions, we obtain

$$
T_{\text {(int) }}^{\mu \nu}=\Lambda_{g} g^{\mu \nu}, \quad \mathcal{T}_{\text {(int) }}^{\mu \nu}=\Lambda_{f} f^{\mu \nu},
$$

thus, the constants $\Lambda_{g}$ and $\Lambda_{f}$ are effective cosmological constants for the $g$-spacetime and the $f$-spacetime, respectively. In what follows, we assume

$$
\Lambda_{g}=\Lambda_{f}=0,
$$

because we are interested not in dark energy but in dark matter. The equations for the homothetic spacetime are exactly reduced into those in GR which indicates that the homothetic solution contains only the massless graviton modes. The degrees of freedom of the massive graviton mode do not exist in the homothetic solution.

\section{ENERGY-MOMENTUM TENSOR OF COHERENT GRAVITONS}

In this section, we derive the effective energy-momentum tensor of the coherently oscillating gravitons focusing on the cosmological situation. General discussion about the energymomentum tensor of gravitons is given in Appendix A.

As is well known in GR, when we discuss some structure produced by high frequency graviational waves, we have to separate the high frequency modes from smoothed background. The length or/and time scale associated with the gravitational waves should be sufficiently shorter than the typical scale of the smooth background [3,4]. Under this setting, the energy-momentum tensor of gravitational waves is defined by the nonlinear terms of the perturbed Einstein equation averaged over a length or/and time scale. We then obtain the propagating equation for the gravitational waves and the Einstein equation for the background including the backreaction from gravitational waves. We shall apply this procedure to the cosmological setting with the coherently oscillating massive gravitons. In the coherent case, we have to take care which we perform a spatial average or a time average.

We consider the homogeneous universe with tiny metric perturbations

$$
\begin{aligned}
& g_{\mu \nu}=g_{\mu \nu}^{(\text {hom })}(t)+\delta g_{\mu \nu}^{(\mathrm{inh})}(t, \mathbf{x}), \\
& f_{\mu \nu}=f_{\mu \nu}^{(\mathrm{hom})}(t)+\delta f_{\mu \nu}^{(\mathrm{inh})}(t, \mathbf{x}),
\end{aligned}
$$

where $g_{\mu \nu}^{\text {(hom })}, f_{\mu \nu}^{\text {(hom) }}$ are the metrics of the homogeneous spacetime and $\delta g_{\mu \nu}^{(\mathrm{inh})}, \delta f_{\mu \nu}^{(\mathrm{inh})}$ represent the inhomogeneous perturbations. Since we are interested in the coherent gravitons, the time coordinate has to be appropriately chosen in order that the $t=$ constant hypersurfaces are given by almost homogeneous spaces. Then, on each hypersurface, the homogeneous parts can be obtained by

$$
g_{\mu \nu}^{(\text {hom })}=\left\langle g_{\mu \nu}\right\rangle_{V}, \quad f_{\mu \nu}^{(\text {hom })}=\left\langle f_{\mu \nu}\right\rangle_{V},
$$

where $\langle\cdots\rangle_{V}$ is the spatial average where the averaged length scale is assumed to be much lager than the scale of the inhomogeneities. The dynamics of the homogeneous spacetime in bigravity was studied in [22]. Up to the linear perturbation theory, one may directly analyze the dynamics of the perturbations under the ansatz (3.1). In the present paper, however, we consider another separation of the metrics rather than (3.1). We first summarize the strategy of our calculations and the explicit analysis are given in Sec. IV and Sec. V.

The bigravity theory contains two types of dynamical degrees of freedom, the massless graviton and the massive graviton. First, we separate the metrics $g_{\mu \nu}$ and $f_{\mu \nu}$ into the massless mode and the massive mode. Up to the linear perturbations around the homothetic background, we can introduce the mass eigenstates of the gravitons. However, the definitions of the massless mode and the massive mode of the metrics would be ambiguous in the nonlinear orders in which the gravitons are no longer diagonalized. (see discussions in $[28,42])$. Nevertheless, the massless mode and the massive mode are still meaningful if the perturbative expansion is viable. Therefore, we only consider the situation that the spacetimes are well approximated by the homothetic solution.

We focus on the late stage of the Universe such that

$$
m^{2} \gg H^{2}
$$

where $H$ is the Hubble expansion rate in which the massive graviton has too heavy mass to be excited. Hence, the amplitude of the massive graviton is suppressed and then the metrics $g_{\mu \nu}$ and $f_{\mu \nu}$ are approximated by the homothetic solution (We recall that the homothetic solution give a spacetime without the excitation of the massive graviton). We then perturbatively treat the massive mode $g_{\mu \nu}^{\text {(massive) }}$ which is defined by the difference between two metrics 
TABLE I. The separations of the metric tensors.

\begin{tabular}{lcc}
\hline \hline & Low-frequency & High-frequency \\
\hline Massless mode $g_{\mu \nu}^{\text {(massless) }}$ & $\stackrel{(0)}{g \nu}_{\mu \nu}+\delta g_{\mu \nu}$ & $h_{\mu \nu} / M_{\mathrm{pl}}$ \\
Massive mode $g_{\mu \nu}^{\text {(massive) }}$ & $M_{\mu \nu}=\bar{M}_{\mu \nu}+\delta M_{\mu \nu}$ & $\varphi_{\mu \nu} / M_{G}=\bar{\varphi}_{\mu \nu} / M_{G}+\delta \varphi_{\mu \nu} / M_{G}$ \\
Homogeneous mode $g_{\mu \nu}^{(\text {hom })}$ & $\bar{g}_{\mu \nu}+\bar{M}_{\mu \nu}$ & $\bar{\varphi}_{\mu \nu} / M_{G}$ \\
Inhomogeneous mode $\delta g_{\mu \nu}^{(\mathrm{inh})}$ & $\delta g_{\mu \nu}+\delta M_{\mu \nu}$ & $h_{\mu \nu} / M_{\mathrm{pl}}+\delta \varphi_{\mu \nu} / M_{G}$ \\
\hline \hline
\end{tabular}

$$
g_{\mu \nu}^{(\text {massive })}=\frac{\alpha}{1+\alpha}\left(g_{\mu \nu}-\xi_{0}^{-2} f_{\mu \nu}\right)
$$

where $\alpha:=\xi_{0}^{2} \kappa_{g}^{2} / \kappa_{f}^{2}$ and we assume $\left|g_{\mu \nu}^{\text {(massive) }}\right| \ll 1$. On the other hand, the massless mode is given by

$$
g_{\mu \nu}^{(\text {massless })}=\frac{1}{1+\alpha}\left(g_{\mu \nu}+\alpha \xi_{0}^{-2} f_{\mu \nu}\right) .
$$

As a result, the metrics $g_{\mu \nu}$ and $f_{\mu \nu}$ can be decomposed into the massless mode and the massive mode as follows:

$$
\begin{aligned}
& g_{\mu \nu}=g_{\mu \nu}^{(\text {massless })}+g_{\mu \nu}^{(\text {massive })} \\
& f_{\mu \nu}=\xi_{0}^{2}\left(g_{\mu \nu}^{(\text {massless })}-\alpha^{-1} g_{\mu \nu}^{(\text {massive })}\right) .
\end{aligned}
$$

We further decompose the massless and the massive modes into the low-frequency modes and the highfrequency modes, respectively:

$$
\begin{aligned}
g_{\mu \nu}^{(\text {massless })} & =\stackrel{(0)}{g \nu}_{\mu \nu}+\frac{h_{\mu \nu}}{M_{\mathrm{pl}}}, \\
g_{\mu \nu}^{(\text {massive })} & =M_{\mu \nu}+\frac{\varphi_{\mu \nu}}{M_{G}},
\end{aligned}
$$

where the high-frequency modes $h_{\mu \nu}$ and $\varphi_{\mu \nu}$ are normalized by two mass scales

$$
M_{\mathrm{pl}}:=\frac{\xi_{0} \bar{\kappa}}{\kappa_{g} \kappa_{f}}, \quad M_{G}:=\frac{\bar{\kappa}}{\kappa_{g}^{2}}=\frac{M_{\mathrm{pl}}}{\alpha^{1 / 2}}
$$

with $\bar{\kappa}^{2}=\kappa_{g}^{2}+\xi_{0}^{-2} \kappa_{f}^{2}$. The low-frequency modes are defined by

$$
\stackrel{(0)}{g_{\mu \nu}}=\left\langle g_{\mu \nu}^{(\text {massless })}\right\rangle_{T}, \quad M_{\mu \nu}=\left\langle g_{\mu \nu}^{(\text {massive })}\right\rangle_{T}
$$

where $\langle\cdots\rangle_{T}$ is the time average. ${ }^{1}$ over some time interval $T$ which is assumed to be

$$
m^{-1} \ll T \ll H^{-1}
$$

\footnotetext{
${ }^{1}$ Alternatively, the low-frequency projection operator can be the oscillation average, i.e., the time average over one coherent oscillation $T=2 \pi / m$.
}

Then, the metric tensors $g_{\mu \nu}$ and $f_{\mu \nu}$ are divided into four components: $\stackrel{(0)}{g}_{\mu \nu}, M_{\mu \nu}, h_{\mu \nu}$ and $\varphi_{\mu \nu}$. The meaning of each variables is summarized in Table I.

We briefly mention the relation between two separations (3.1) and (3.7). The variables $\stackrel{g}{\mu \nu}_{\mu \nu}, M_{\mu \nu}, h_{\mu \nu}$ and $\varphi_{\mu \nu}$ are divided into the homogeneous parts and the inhomogeneous parts

$$
\begin{aligned}
\stackrel{(0)}{g}_{\mu \nu} & =\bar{g}_{\mu \nu}(t)+\delta g_{\mu \nu}(t, \mathbf{x}), \\
M_{\mu \nu} & =\bar{M}_{\mu \nu}(t)+\delta M_{\mu \nu}(t, \mathbf{x}), \\
h_{\mu \nu} & =h_{\mu \nu}(t, \mathbf{x}), \\
\varphi_{\mu \nu} & =\bar{\varphi}_{\mu \nu}(t)+\delta \varphi_{\mu \nu}(t, \mathbf{x}),
\end{aligned}
$$

where the homogeneous parts are defined via the spatial average $\langle\cdots\rangle_{V}$ as with (3.2). We then obtain

$$
\begin{gathered}
g_{\mu \nu}^{(\mathrm{hom})}=\bar{g}_{\mu \nu}+\bar{M}_{\mu \nu}+\frac{\bar{\varphi}_{\mu \nu}}{M_{G}}, \\
\delta g_{\mu \nu}^{(\mathrm{inh})}=\delta g_{\mu \nu}+\delta M_{\mu \nu}+\frac{h_{\mu \nu}}{M_{\mathrm{pl}}}+\frac{\delta \varphi_{\mu \nu}}{M_{G}} .
\end{gathered}
$$

It is worth noting that

$$
\left\langle h_{\mu \nu}\right\rangle_{V}=0, \quad\left\langle\varphi_{\mu \nu}\right\rangle_{V}=\bar{\varphi}_{\mu \nu} \neq 0,
$$

because $h_{\mu \nu}$ is massless while $\varphi_{\mu \nu}$ is massive. The zero momentum mode of the massless graviton cannot be highfrequency whereas that of the massive graviton can be high-frequency due to the coherent oscillation. Since we have assumed that the configuration of the fields are almost homogeneous, the massive graviton is dominated by the zero momentum mode $\bar{\varphi}_{\mu \nu}$, that is,

$$
\left|\bar{\varphi}_{\mu \nu}\right| \gg\left|\delta \varphi_{\mu \nu}\right|
$$

We call this configuration of $\varphi_{\mu \nu}$ the massive graviton condensate because a large fraction of $\varphi_{\mu \nu}$ occupies the single zero momentum state $\bar{\varphi}_{\mu \nu}$.

In the separation (3.1), the "backgrounds," i.e., the homogeneous modes $g_{\mu \nu}^{\text {(hom) }}$ and $f_{\mu \nu}^{\text {(hom) }}$, are obtained by the spatial average whereas the "background" in (3.7), i.e., 
the low-frequency massless mode $g_{\mu \nu}^{(0)}$, is given by the time average and then it can be inhomogeneous. An advantage of the separation (3.7) is that the high-frequency "perturbations" $h_{\mu \nu}$ and $\varphi_{\mu \nu}$ can be treated as tensor fields, propagating on the low-frequency "background" ${ }_{g}^{(0)}$, with well-defined energy-momentum tensors.

The amplitude of the massive graviton is small so we have the inequalities

$$
\left|M_{\mu \nu}\right|, \quad\left|\varphi_{\mu \nu}\right| / M_{G} \ll\left|g_{\mu \nu}^{(0)}\right| .
$$

The amplitude of $h_{\mu \nu} / M_{\mathrm{pl}}$ is also small since $h_{\mu \nu}$ is a part of the inhomogeneity. As a result, we have three small quantities $M_{\mu \nu}, h_{\mu \nu}$ and $\varphi_{\mu \nu}$ which can be treated as the tensors with respect to the "background" metric $\stackrel{(0)}{\mu \nu}$. We adopt the notation such that the suffices on $M_{\mu \nu}, h_{\mu \nu}$ and $\varphi_{\mu \nu}$ are raised and lowered by $\stackrel{(0)}{\mu \nu}_{\mu \nu}$. However, the inequality (3.16) does not suggest that the backreaction of $\varphi_{\mu \nu}$ to $g_{\mu \nu}^{(0)}$ is also small. The orders of magnitude of the Einstein tensor of $\stackrel{(0)}{g \nu}_{\mu \nu}$ and the energy-momentum tensor of $\varphi_{\mu \nu}$, which we denote $\stackrel{(0)}{G}_{\mu \nu}$ and $T_{G}^{\mu \nu}$, are estimated as

$$
\left|\stackrel{(0)}{G_{\mu \nu}}\right| \sim H^{2}, \quad\left|T_{G}^{\mu \nu}\right| \sim m^{2}\left|\varphi_{\mu \nu}^{2}\right|,
$$

where $T_{G}^{\mu \nu}$ is explicitly defined by (3.22) below. Thus, if $\left|\varphi_{\mu \nu}\right| / M_{G} \sim H / m \ll 1$, the massive graviton $\varphi_{\mu \nu}$ can be a dominant component of the Universe. In what follows, we assume the massive graviton is the dominant component.

Just for simplicity, we consider the case

$$
h_{\mu \nu}=0 \text {. }
$$

This is a specific case, but this assumption is reasonable for our interest since the massless gravitons, i.e., the gravitational waves, are sub-dominant in the Universe. To discuss the dynamics of the Universe, the effect of the massless gravitons can be ignored.

To discuss dynamics of the massive graviton condensate $\varphi_{\mu \nu}$, it is sufficient to include the leading and subleading contributions associated with the adiabatic expansion in terms of $\mathrm{m}^{-1}{ }^{2}$ Up to subleading order, we can ignore $M_{\mu \nu}$ since the amplitude of $M_{\mu \nu}$ is suppressed by $\mathrm{m}^{-2}$ which is the sub-subleading order (see Appendix B). The low-frequency massive mode $M_{\mu \nu}$ gives only negligible contributions.

\footnotetext{
${ }^{2}$ More precisely, we use the dimensionless parameter $H / m$ for the adiabatic expansion. We just refer to $m^{-1}$ as the order of the expansion.
}

Ignoring $h_{\mu \nu}$ and $M_{\mu \nu}$, the $g$-spacetime metric is given by

$$
g_{\mu \nu}=\stackrel{(0)}{g}_{\mu \nu}+\frac{\varphi_{\mu \nu}}{M_{G}} .
$$

The equations for $\stackrel{(0)}{g \nu}_{\mu \nu}$ is given by the time-averaged Einstein equation

$$
\stackrel{(0)}{G^{\mu \nu}}=\frac{1}{M_{\mathrm{pl}}^{2}}\left(\stackrel{(0)}{T^{\mu \nu}}+\left\langle T_{G}^{\mu \nu}\right\rangle_{T}\right),
$$

where the "effective" energy-momentum tensors of the (0) matter $T^{\mu \nu}$ and that of the massive gravitons $T_{G}^{\mu \nu}$ are defined by the relation

$$
\left\langle T_{\mu \nu}-\frac{1}{2} g_{\mu \nu} T\right\rangle_{T}=T_{\mu \nu}^{(0)}-\frac{1}{2} g_{\mu \nu}^{(0)} T_{\alpha \beta}^{(0)} g^{(0)},
$$

and

$$
\begin{aligned}
T_{G}^{\mu \nu}= & -\left(\stackrel{(0)}{g^{\mu \alpha}} g^{\nu \beta}-\frac{1}{2} g^{\mu \nu} g^{\alpha \beta}\right) \delta R_{\alpha \beta}^{(2)}[\varphi] \\
& -\frac{m_{\mathrm{eff}}^{2}}{8}\left(4 \varphi^{\mu \alpha} \varphi_{\alpha}^{\nu}-\stackrel{(0)}{ }^{\mu \nu} \varphi^{\alpha \beta} \varphi_{\alpha \beta}\right)+\mathcal{O}\left(\varphi^{3}\right) .
\end{aligned}
$$

The equation of motion for $\varphi_{\mu \nu}$ is

$$
\begin{aligned}
& \delta R_{\mu \nu}^{(1)}[\varphi]+\frac{m_{\mathrm{eff}}^{2}}{4}\left(2 \varphi_{\mu \nu}+\varphi^{\alpha}{ }_{\alpha}{ }_{\mu \nu}^{(0)}\right)+\left\langle\delta \mathcal{E}_{\mu \nu}\right\rangle_{\mathrm{high}}+\mathcal{O}\left(\varphi^{4}\right) \\
& =\frac{1}{M_{G}}\left\langle T_{\mu \nu}-\frac{1}{2} g_{\mu \nu} T\right\rangle_{\mathrm{high}},
\end{aligned}
$$

where the effective graviton mass $m_{\text {eff }}$ is defined by

$$
m_{\mathrm{eff}}^{2}:=m^{2} \frac{\bar{\kappa}^{2}}{\kappa^{2}}\left(b_{1} \xi_{0}+2 b_{2} \xi_{0}^{2}+b_{3} \xi_{0}^{3}\right)
$$

and $\delta \mathcal{E}_{\mu \nu}^{(2)}$ include the terms of quadratic in $\varphi_{\mu \nu}$ which is explicitly given by

$$
\begin{aligned}
\delta \mathcal{E}_{\mu \nu}^{(2)}= & \frac{\alpha-1}{\alpha^{1 / 2} M_{\mathrm{pl}}} \delta R_{\mu \nu}^{(2)}[\varphi]+\frac{m_{\mathrm{eff}}^{2}}{16 \alpha^{1 / 2} M_{\mathrm{pl}}} \\
& \times\left[3(1-\alpha) \stackrel{(0)}{\mu \nu}_{\mu \beta} \varphi_{\alpha \beta} \varphi^{\alpha \beta}+4\left\{\left(1-\beta_{2}\right) \alpha-\beta_{2}\right\} \varphi_{\mu \nu} \varphi^{\alpha}{ }_{\alpha}\right. \\
& \left.+2\left\{\left(1+2 \beta_{2}\right) \alpha-\left(3-2 \beta_{2}\right) \alpha\right\} \varphi_{\mu}^{\alpha} \varphi_{\nu \alpha}\right],
\end{aligned}
$$

with

$$
\beta_{2}=\frac{b_{2} \xi_{0}^{2}+b_{3} \xi_{0}^{3}}{b_{1} \xi_{0}+2 b_{2} \xi_{0}^{2}+b_{2} \xi_{0}^{3}} .
$$

The symbol $\langle\cdots\rangle_{\text {high }}$ denotes a high-frequency projection operator which is given by 


$$
\langle X\rangle_{\text {high }}=X-\langle X\rangle_{T}
$$

for a quantity $X$. The functionals $\delta R_{\mu \nu}^{(1)}$ and $\delta R_{\mu \nu}^{(2)}$ are the first order and the second order of the perturbed Ricci curvatures which are explicitly shown in Appendix A.

The amplitude of the coherent oscillation decreases due to the Hubble friction which finally cause the decreasing of the energy density of the massive graviton condensate. To solve (3.23), we have to retain terms of linear in first derivatives of the metric $g_{\mu \nu}$. ${ }^{3}$ On the other hand, we may ignore terms of higher orders of derivatives of $g_{\mu \nu}^{(0)}$ which are sub-subleading order contributions; thus, the covariant derivatives commute

$$
\stackrel{(0)}{\nabla_{[\alpha}} \stackrel{(0)}{\nabla_{\beta]}} \varphi_{\mu \nu} \approx 0
$$

where $\stackrel{(0)}{\nabla}_{\mu}$ is the covariant derivative 2 ) with respect to $\stackrel{(0)}{g}_{\mu \nu}$.

Note that the quadratic terms $\delta \mathcal{E}_{\mu \nu}$ cannot be ignored. For the homogeneous ansatz, the Friedmann equation schematically reads

$$
H^{2} \sim \frac{1}{M_{\mathrm{pl}}^{2}} m^{2} \varphi^{2}+\mathcal{O}\left(\varphi^{3}\right)
$$

when the massive graviton is the dominant component of the Universe (see Sec. IV for the explicit expressions). The quadratic term in (3.23) is then

$$
\delta \mathcal{E}_{\mu \nu}^{(2)} \sim \frac{m^{2}}{M_{\mathrm{pl}}} \varphi^{2} \sim H m \varphi,
$$

which yields a comparable effect to the first derivative of the metric. Therefore, we should solve the nonlinear differential equation (3.23) to discuss the dynamics of the coherent massive graviton, in general. However, we assume the $Z_{2}$ symmetry for the self-interactions of the massive graviton: the interaction terms are invariant under the $Z_{2}$ transformation $\varphi_{\mu \nu} \rightarrow-\varphi_{\mu \nu}$, it prohibits appearance (2) of $\delta \mathcal{E}_{\mu \nu}$ and then the basic equations become much simpler. The $Z_{2}$ symmetry is realized by supposing the symmetry of the gravitational action under the replacement

$$
g_{\mu \nu} \leftrightarrow f_{\mu \nu},
$$

which is realized when

\footnotetext{
${ }^{3}$ When $\varphi_{\mu \nu}$ is treated as a particle, the graviton may be treated as a freely propagating on the flat background since the particle do not feel the effect of the curvature in a small scale. The particle dark matter scenario in bigraivty has been discussed in $[17,27,28]$.
}

$$
\kappa_{g}=\kappa_{f}, \quad b_{i}=b_{4-i}, \quad(i=0-4) .
$$

In this case, $\xi_{0}=1$ is always a solution to the equation (2.9). For the branch $\xi_{0}=1$, clearly from the definition of the massive mode, the symmetry (3.31) realizes the $Z_{2}$ symmetry of the massive graviton. Indeed, the parameters (3.32) yield $\alpha=1, \beta_{2}=1 / 2$ and then

$$
\delta \mathcal{E}_{\mu \nu}^{(2)} \equiv 0 .
$$

As a result, the equation for the massive mode is linear since the cubic terms can be ignored for our calculations. ${ }^{4}$

By using the normalization of the mass parameter $m$, we can always set

$$
b_{1}+2 b_{2}+b_{3}=1
$$

in which we obtain

$$
m_{\mathrm{eff}}=m
$$

thus, the mass parameter $m$ indeed corresponds to the graviton mass in the branch $\xi_{0}=1$. We shall use this normalization in what follows. Combining this normalization with (2.13) and (3.32), the coupling constants $b_{i}$ are expressed by only $b_{2}$ as

$$
b_{0}=b_{4}=b_{2}-2, \quad b_{1}=b_{3}=\frac{1}{2}-b_{2} .
$$

The effective energy-momentum tensor $T_{\mu \nu}^{(0)}$ is obtained from the smoothing of the true energy-momentum tensor $T_{\mu \nu}$. Even if we assume the true energy-momentum tensor is conserved, i.e., $\nabla_{\mu}^{(g)} T^{\mu \nu}=0$, the smoothed energymomentum tensor is not conserved, in general, since the energy of the matter can be converted to the one of the graviton and vice versa via the equation (3.23). The contracted Bianchi identity of (3.20) reads the smoothed total energy-momentum tensor is conserved:

$$
\stackrel{(0)}{\nabla}_{\mu}\left(\stackrel{(0)}{T^{\mu \nu}}+\left\langle T_{G}^{\mu \nu}\right\rangle_{T}\right)=0 .
$$

However, in the late stage of the Universe, the massive gravitons must be decoupled from the matter due to the weakness of the gravitational interaction, i.e.,

$$
\frac{1}{M_{G}}\left\langle T_{\mu \nu}-\frac{1}{2} g_{\mu \nu} T\right\rangle_{\text {high }} \approx 0 .
$$

\footnotetext{
${ }^{4}$ The parameters $\alpha$ and $\beta_{2}$ do not appear at linear order except for the right-hand side of Eq. (3.23) (we note $M_{G}=\alpha^{-1 / 2} M_{\mathrm{pl}}$ ). As a result, the existence of the $Z_{2}$ symmetry (3.32) does not change the theory up to the linear order except for the coupling strength to the matter.
} 
Then, the energy-momentum tensors are individually conserved:

$$
\begin{gathered}
\stackrel{(0)}{\nabla_{\mu}} \stackrel{(0)}{T^{\mu \nu}} \approx 0, \\
\stackrel{(0)}{\nabla}_{\mu}\left\langle T_{G}^{\mu \nu}\right\rangle_{T} \approx 0 .
\end{gathered}
$$

The conservation of $T_{G}^{\mu \nu}$ is directly confirmed by using the equation of motion. For the freely propagating gravitons (3.38), the equation (3.23) is reduced into

$$
\begin{aligned}
& \left(\stackrel{(0)}{\nabla_{\alpha}} \stackrel{(0)}{\nabla^{\alpha}}-m_{\mathrm{eff}}^{2}\right) \varphi_{\mu \nu} \approx 0, \\
& \stackrel{(0)}{\nu}_{\mu} \varphi^{\mu \nu} \approx 0, \quad \varphi^{\alpha}{ }_{\alpha} \approx 0 .
\end{aligned}
$$

Using these equations, one can find

$$
\stackrel{(0)}{\nabla}_{\mu} T_{G}^{\mu \nu} \approx 0
$$

which is a sufficient condition on the conservation of the graviton energy-momentum tensor (3.40). We notice, however, that two conservations (3.40) and (3.43) are not equivalent since (3.40) reads that the smoothed quantity of $T_{G}^{\mu \nu}$ is conserved. Equation (3.40) has information only about macroscopic behavior of $\varphi_{\mu \nu}$ while Eq. (3.43) [or (3.41) and (3.42)] involves information about microscopic behavior.

In the following sections, we will show that there exists a solution such that $\varphi_{\mu \nu}$ behaves as dark matter which explains not only the background dynamics of the Universe but also the structure formation. Since we have assumed the inhomogeneities are smaller than the homogeneous modes, we shall linearize the expressions in terms of the inhomogeneities. For instance, the graviton energymomentum tensor is expressed as

$$
T_{G}^{\mu \nu}=\bar{T}_{G}^{\mu \nu}(t)+\delta T_{G}^{\mu \nu}(t, \mathbf{x}),
$$

with, in order of magnitude,

$$
\left|\delta T_{G}^{\mu \nu}(t, \mathbf{x})\right| \sim\left|\bar{\varphi}_{\alpha \beta} \partial^{2} \delta \varphi_{\mu \nu}\right| .
$$

\section{MASSIVE GRAVITON CONDENSATE AS DARK MATTER}

In this section, we consider the homogeneous mode $\bar{g}_{\mu \nu}$ and $\bar{\varphi}_{\mu \nu}$. We assume the flat Friedmann-LemaitreRobertson-Walker (FLRW) background

$$
\bar{g}_{\mu \nu} d x^{\mu} d x^{\nu}=-d t^{2}+a^{2}\left[d x^{2}+d y^{2}+d z^{2}\right],
$$

and a simple ansatz for $\bar{\varphi}_{\mu \nu}$

$$
\bar{\varphi}_{\mu \nu}=\operatorname{diag}\left[0,4 a^{2} \bar{\varphi},-2 a^{2} \bar{\varphi},-2 a^{2} \bar{\varphi}\right],
$$

where $a$ and $\bar{\varphi}$ are functions of $t$. Note that the ansatz (4.2) trivially satisfies the constraints (3.42).

The massive graviton originally appears from the metric perturbations. The present set up (4.2) corresponds to considering the axisymmetric Bianchi type I universe in bigravity which we will detail in Appendix B (see [22] for more details). One may worry about that $\bar{g}_{\mu \nu}$ should be also given by the axisymmetric Bianchi type I universe rather than FLRW universe (4.1). However, as we will see just below, the averaged graviton energy-momentum tensor is indeed isotropic and (4.2) is consistent with (4.1) (see also [43]). Furthermore, even if one replaces (4.1) with the Bianchi-type universe, its anisotropy decreases as $a^{-6}$ and then the anisotropic part in $\bar{g}_{\mu \nu}$ is quickly ignored.

The equation (3.41) including up to the first derivatives of the metric reads

$$
\ddot{\ddot{\varphi}}+3 H \dot{\bar{\varphi}}+m^{2} \bar{\varphi}=0,
$$

where $H=\dot{a} / a$. By using (4.3), we find

$$
\begin{aligned}
& \bar{T}_{G}^{t t}=3\left(\dot{\bar{\varphi}}^{2}+m^{2} \bar{\varphi}^{2}\right), \\
& \bar{T}_{G}^{x x}=a^{-2}\left(m^{2} \bar{\varphi}^{2}-\dot{\bar{\varphi}}^{2}\right), \\
& \bar{T}_{G}^{y y}=\bar{T}_{G}^{z z}=7 a^{-2}\left(m^{2} \bar{\varphi}^{2}-\dot{\bar{\varphi}}^{2}\right),
\end{aligned}
$$

and other components are zero. The approximative solution to $(4.3)$ is

$$
\bar{\varphi}=\frac{\bar{\varphi}_{0}}{a^{3 / 2}} \cos \left[m t+\theta_{0}\right]
$$

where $\bar{\varphi}_{0}$ and $\theta_{0}$ are integration constants. Since the initial phase $\theta_{0}$ is not important for the discussion, we set $\theta_{0}=0$. After averaging over the time interval $T \gg m^{-1}$, the graviton energy-momentum tensor is calculated as

$$
\left\langle\bar{T}_{G}^{\mu \nu}\right\rangle_{T}=\operatorname{diag}\left[\bar{\rho}_{G}, 0,0,0\right]
$$

where the energy of the massive graviton condensate is

$$
\bar{\rho}_{G}=\frac{3}{a^{3}} m^{2} \bar{\varphi}_{0}^{2}
$$

Therefore, the massive graviton condensate behaves as a dust fluid. This guarantees the ansatz (4.1).

To explain the abundance of dark matter, the amplitude of $\bar{\varphi}$ is required to be

$$
\bar{\varphi} \sim M_{\mathrm{pl}} \frac{H_{0}}{m},
$$


where $H_{0}$ is the present Hubble parameter. Since the physical spacetime is given by (3.19), the Universe is filled with the coherent "gravitational waves" $\varphi_{\mu \nu} / M_{\mathrm{pl}}$ whose dimensionless amplitude and the frequency are

$$
\begin{gathered}
\left|\varphi_{\mu \nu} / M_{\mathrm{pl}}\right| \sim 10^{-29}\left(\frac{10^{-4} \mathrm{eV}}{m}\right), \\
f \sim 10^{11}\left(\frac{m}{10^{-4} \mathrm{eV}}\right) \mathrm{Hz} .
\end{gathered}
$$

The oscillations have too small amplitude and too high frequency and thus there should be no constraint on the existence of $\varphi_{\mu \nu}$ at present.

\section{COSMIC STRUCTURE FORMATION}

To study the cosmic structure formation, we then introduce small inhomogeneity to the metric and the massive graviton $\varphi_{\mu \nu}$. Note that the low-frequency "background" ${ }_{g \nu}^{(0)}$ is not necessary to be homogeneous (see (3.11). We treat ${ }_{g \nu}^{(0)}$ including the inhomogeneity $\delta g_{\mu \nu}$ as the low-frequency massless mode which is verified as long as the momentum of the inhomogeneity is smaller than the graviton mass:

$$
\frac{k^{2}}{a^{2}} \ll m^{2}
$$

where $k$ is the comoving momentum of the inhomogeneity defined by (5.16) later.

For the calculations, we use the adiabatic expansion in terms of the graviton mass inverse $m^{-1}$ (see Section 2.5 in [44] for calculations in the case of the scalar condensate and [45] for the vector case). We set the orders of both $\bar{g}_{\mu \nu}(t)$ and $\delta g_{\mu \nu}(t, \mathbf{x})$ as $\mathcal{O}\left(m^{0}\right)$. Since the time derivatives acting on the low-frequency modes do not change the order of magnitude $m^{-1}$, i.e., $\partial / \partial t=\mathcal{O}\left(m^{0}\right)$, the Friedmann equation leads to $\bar{\rho}_{G}=\mathcal{O}\left(m^{0}\right)$. Hence, the homogeneous mode of the massive graviton $\bar{\varphi}_{\mu \nu}$ is of order $\mathcal{O}\left(\mathrm{m}^{-1}\right)$. On the other hand, the amplitude of the inhomogeneous mode of the massive graviton $\delta \varphi_{\mu \nu}$ is of order $\mathcal{O}\left(m^{0}\right)$ as we will show later.

To evaluate the inhomogeneous parts of $T_{G}^{\mu \nu}$ the coherent background including the subleading order is required which is given by

$$
\bar{\varphi}=\bar{\varphi}_{1} \cos [m t]+\bar{\varphi}_{2} \sin [m t]+\mathcal{O}\left(m^{-3}\right),
$$

where $\bar{\varphi}_{1}, \bar{\varphi}_{2}$ are slowly varying functions with

$$
\bar{\varphi}_{1}=\frac{\bar{\varphi}_{0}}{a^{3 / 2}}=\mathcal{O}\left(m^{-1}\right), \quad \bar{\varphi}_{2}=\mathcal{O}\left(m^{-2}\right),
$$

To determine the explicit form of $\bar{\varphi}_{2}$ we need to solve the equation (4.3) up to the order $\mathcal{O}\left(\mathrm{m}^{-1}\right)$; however, the equation (4.3) is valid up to the order $\mathcal{O}\left(\mathrm{m}^{0}\right)$. Nevertheless, the explicit form of $\bar{\varphi}_{2}$ is not necessary to evaluate $\delta T_{G}^{\mu \nu}$.

Since we take the adiabatic expansion in terms of not $\mathrm{k} / \mathrm{m}$ but $\mathrm{H} / \mathrm{m}$, the spatial derivatives do or do not change the order $\mathrm{m}^{-1}$ depending on the scales of the inhomogeneities. For the large scales such that

$$
\frac{k^{2}}{a^{2}} \ll m H
$$

the spatial derivatives acting on the variables do not change the order of $m^{-1}$, i.e.,

$$
k^{2}=\mathcal{O}\left(m^{0}\right)
$$

in which the consistency of the Einstein equation leads to

$$
\left\langle\delta T_{G}^{\mu \nu}\right\rangle_{T}=\mathcal{O}\left(m^{0}\right) .
$$

On the other hand, for the small scales

$$
\frac{k^{2}}{a^{2}} \gtrsim m H
$$

the order of $k$ is

$$
k^{2}=\mathcal{O}(m)
$$

Then, the graviton energy-momentum tensor is evaluated as

$$
\left\langle\delta T_{G}^{\mu \nu}\right\rangle_{T}=\mathcal{O}\left(m^{1}\right) .
$$

As we will show, this classification of the scales corresponds to the scales beyond or below the Jeans scale.

The graviton mass should be $m \gtrsim 10^{-4} \mathrm{eV}$ since we have not detected any deviations from the Newtonian gravitational law in the laboratory scales. ${ }^{5}$ In that case, the scale $a / k \sim(m H)^{-1 / 2}$ is quite small compared with the structures of the Universe. Therefore, the case (5.7) is irrelevant to the cosmic structure formation. Nevertheless, we shall discuss both scales (5.4) and (5.7), for completeness. Furthermore, the discussion about the small scale (5.7) will make the properties of the massive graviton condensate clear.

We have ignored sub-subleading terms to derive the equations (3.41) and (3.42). Supposing $\delta \varphi_{\mu \nu}$ is of order $\mathcal{O}\left(m^{0}\right)$, these equation showing the order of errors explicitly are written as

\footnotetext{
${ }^{5}$ The constraints are obtained from the linearized theory. The precise constraints on the nonlinear bigravity theory are subject to discussion.
} 


$$
\begin{gathered}
\left(\stackrel{(0)}{\nabla} \stackrel{(0)}{\nabla}^{\alpha}-m^{2}\right) \varphi_{\mu \nu}=0+\mathcal{O}\left(m^{0}\right), \\
\stackrel{(0)}{\nabla}_{\mu} \varphi^{\mu \nu}=0+\mathcal{O}\left(m^{-1}\right) \\
\varphi_{\alpha}^{\alpha}=0+\mathcal{O}\left(m^{-2}\right) .
\end{gathered}
$$

The corrections to the equations come from the approximations Eqs. (3.28) and (3.38) as well as the nonlinear quantities of $\varphi_{\mu \nu}$. It is worth noting that, as long as the expressions are linear in inhomogeneities, the accuracy of the calculations are same in both cases (5.4) and (5.7). As for the graviton energy-momentum tensor, although we have ignored higher-order corrections to $\delta T_{G}^{\mu \nu}$ such as $m^{2} \bar{\varphi}^{3} \delta \varphi$, they are of order $\mathcal{O}\left(m^{-1}\right)$. Up to the order $\mathcal{O}\left(m^{0}\right)$, the higher-order terms are negligible.

In the standard cosmological perturbation theory, general perturbations can be decomposed into scalar-type, vectortype, and tensor-type perturbations and they are decoupled at the linear order due to the spatial homogeneity and isotropy of the background. In the present case, the background configuration $\bar{\varphi}_{\mu \nu}$ breaks the spatial rotational symmetry and then the different modes could couple in the scalar-vector-tensor decomposition (see [46] for perturbations around the anisotropic universe). However, there still exists the rotational symmetry in the $y-z$ plane. The perturbations can be decomposed into the even parity perturbations and the odd parity perturbations associated with the rotation in the $y-z$ plane:

$$
\begin{gathered}
\delta g_{\mu \nu}=\delta g_{\mu \nu}^{(\text {even })}+\delta g_{\mu \nu}^{\text {(odd) }}, \\
\delta \varphi_{\mu \nu}=\delta \varphi_{\mu \nu}^{\text {(even) }}+\delta \varphi_{\mu \nu}^{\text {(odd) }}
\end{gathered}
$$

where explicit forms of perturbations are shown in Appendix C. Furthermore, due to the spatial translation symmetry of the background all variables can be transformed into the momentum space, e.g.,

$$
\phi(t, \mathbf{x}) \rightarrow \phi(t) e^{i\left(k_{x} x+k_{y} y+k_{z} z\right)},
$$

and the different momentum modes do not couple. Henceforth, we use variables in the momentum space and the notation

$$
\begin{gathered}
k_{\|}^{2}=k_{y}^{2}+k_{z}^{2}, \\
k^{2}=k_{x}^{2}+k_{y}^{2}+k_{z}^{2} .
\end{gathered}
$$

For the calculations, we decompose the perturbations into the odd parity perturbations and the even parity perturbations. However, to clarify the physical meaning of the results, we shall divide the odd parity perturbations and the even parity perturbations into the scalar-type, the vector-type, and the tensor-type components. We define three-dimensional harmonic scalar $Y_{S}$, vectors $Y_{V}^{i}, \mathcal{Y}_{V}^{i}$, and tensors $Y_{T}^{i j}, \mathcal{Y}_{T}^{i j}$ which satisfy

$$
\begin{array}{ll}
\partial^{2} Y_{S}=-k^{2} Y_{S}, & \\
\partial^{2} Y_{V}^{i}=-k^{2} Y_{V}^{i}, & \partial^{2} \mathcal{Y}_{V}^{i}=-k^{2} \mathcal{Y}_{V}^{i}, \\
\partial^{2} Y_{T}^{i j}=-k^{2} Y_{T}^{i j}, & \partial^{2} \mathcal{Y}_{T}^{i j}=-k^{2} \mathcal{Y}_{T}^{i j},
\end{array}
$$

and

$$
\begin{aligned}
\partial_{i} Y_{V}^{i} & =\partial_{i} \mathcal{Y}_{V}^{i}=0, \quad \partial_{i} Y_{T}^{i j}=\partial_{i} \mathcal{Y}_{T}^{i j}=0, \\
Y_{T i}^{i} & =\mathcal{Y}_{T i}^{i}=0,
\end{aligned}
$$

where $\partial^{2}=\partial_{i} \partial^{i}$ and $i, j=(x, y, z)$. The indices $i, j$ are raised and lowered by $\delta^{i j}$ and $\delta_{i j}$. The quantities $Y_{S}, Y_{V}^{i}, Y_{T}^{i j}$ are even parity quantities associated with the two dimensional rotation in the $y-z$ plane while $\mathcal{Y}_{V}^{i}, \mathcal{Y}_{T}^{i j}$ are odd parity quantities. The suffixes $S, V$, and $T$ are attached to classify the quantities into the scalar, the vector, and the tensor types associated with the three dimensional rotation. We further introduce the quantities as

$$
\begin{gathered}
Y_{S}^{i}=-\frac{1}{k} \partial^{i} Y_{S}, \\
Y_{S}^{i j}=k^{-2}\left(\partial^{i} \partial^{j}-\frac{1}{3} \partial^{k} \partial_{k} \delta^{i j}\right) Y_{S}, \\
Y_{V}^{i j}=-\frac{1}{k} \partial^{(i} Y_{V}^{j)}, \\
\mathcal{Y}_{V}^{i j}=-\frac{1}{k} \partial^{(i} \mathcal{Y}_{V}^{j)},
\end{gathered}
$$

then we obtain nine harmonics which are summarized in Table II.

Using the gauge condition (see Appendix C for details), the perturbations for the low-frequency mode $\delta g_{\mu \nu}$ can be given by

$$
\begin{gathered}
\delta g_{\mu \nu}^{\text {even }}=\left(\begin{array}{cc}
-2 \Phi Y_{S} & -a B_{V} Y_{V j} \\
* & 2 a^{2}\left(\Psi Y_{S} \delta_{i j}+H_{T} Y_{T i j}\right)
\end{array}\right), \\
\delta g_{\mu \nu}^{\text {odd }}=\left(\begin{array}{cc}
0 & -a \mathcal{B}_{V} \mathcal{Y}_{V j} \\
* & 2 a^{2} \mathcal{H}_{T} \mathcal{Y}_{T i j}
\end{array}\right),
\end{gathered}
$$

where $\Phi, \Psi, B_{V}, H_{T}, \mathcal{B}_{V}, \mathcal{H}_{T}$ are functions of $t$.

TABLE II. The classifications of the even parity perturbations and the odd parity perturbations.

\begin{tabular}{lrrr}
\hline \hline & Scalar & Vector & Tensor \\
\hline Even parity & $Y_{S}, Y_{S}^{i}, Y_{S}^{i j}$ & $Y_{V}^{i}, Y_{V}^{i j}$ & $Y_{T}^{i j}$ \\
Odd parity & None & $\mathcal{Y}_{V}^{i}, \mathcal{Y}_{V}^{i j}$ & $\mathcal{Y}_{T}^{i j}$ \\
\hline \hline
\end{tabular}


We will calculate the perturbed graviton energymomentum tensor in the following subsections. In the momentum space, the perturbed graviton energy-momentum tensor is given by the form

$$
\begin{gathered}
\left\langle\delta T_{G}^{(\text {even }) \mu \nu}\right\rangle_{T} \\
=\left(\begin{array}{cc}
\left(\delta \rho_{G}-2 \Phi \bar{\rho}_{G}\right) Y_{S} & a^{-1} \bar{\rho}_{G} v_{(\text {even })}^{j} \\
* & a^{-2}\left[\delta p_{G} Y_{S} \delta^{i j}+\Pi_{(\text {even })}^{i j}\right]
\end{array}\right) \\
\left\langle\delta T_{G}^{\text {(odd) } \mu \nu}\right\rangle_{T}=\left(\begin{array}{cc}
0 & a^{-1} \bar{\rho}_{G} v_{(\text {odd })} \mathcal{Y}_{V}^{j} \\
* & a^{-2} \Pi_{\text {(odd })}^{i j}
\end{array}\right),
\end{gathered}
$$

from which we can read the energy, the velocity, the isotropic pressure, and the anisotropic stress regarding the massive graviton condensate as a fluid. The velocity for the even parity perturbation is decomposed into

$$
v_{(\text {even })}^{i}=v_{S} Y_{S}^{i}+v_{V} Y_{V}^{i},
$$

whereas the anisotropic stresses are decomposed into

$$
\begin{gathered}
\Pi_{\text {(even) }}^{i j}=\pi_{S} Y_{S}^{i j}+\pi_{V} Y_{V}^{i j}+\pi_{T} Y_{T}^{i j}, \\
\Pi_{\text {(odd) }}^{i j}=\pi_{V}^{\text {(odd) }} \mathcal{Y}_{V}^{i j}+\pi_{T}^{\text {(odd) }} \mathcal{Y}_{T}^{i j} .
\end{gathered}
$$

Since calculations for general perturbations are complicated, we just show particular solutions. The generic solutions are given in Appendix C.

First, the odd parity perturbations are not important for the structure formation since these modes contain only the vector-type and the tensor-type perturbations. We discuss only the even-parity perturbations in this section. Furthermore, we find the vector-type components are always decoupled up to the subleading order, whereas the scalar-type and the tensor-type components are coupled in the small scales. Since the vector-type perturbations represent the rotational modes and decay in time, the vector modes are not important. Hence, it is sufficient for the structure formation to consider the even-parity perturbations without the contributions from the vector-type components.

The irrotational solution for the even-parity perturbations can be found under the ansatz

$$
\begin{aligned}
& \delta g_{\mu \nu}(t, \mathbf{k})=\left(\begin{array}{cc}
-2 \Phi Y_{S} & 0 \\
* & 2 a^{2}\left(\Psi Y_{S} \delta_{i j}+H_{T} Y_{T i j}\right)
\end{array}\right), \\
& \delta \varphi_{\mu \nu}(t, \mathbf{k}) \\
& =\left(\begin{array}{ccc}
-2 \phi Y_{S} & -a B Y_{x} & -a C Y_{a} \\
* & 2 a^{2}(\psi+2 \delta \varphi) Y_{S} & 0 \\
* & * & 2 a^{2}(\psi-\delta \varphi) \delta_{a b} Y_{S}
\end{array}\right) \\
& \quad+\mathcal{O}\left(m^{-2}\right),
\end{aligned}
$$

where $a, b=(y, z)$ whose indices are raised and lowered by $\delta_{a b}$ and $Y_{x}$ and $Y_{a}$ are defined by (C3). Since the nondiagonal components of $\delta \varphi_{i j}$ only have decaying modes, we just set $\delta \varphi_{i j}=0$ for $i \neq j$. Note that we do not use the scalar-vector-tensor-type harmonics to represent the components of the massive graviton in order that the expression is written in a clear form. Under the adiabatic expansion, the variables for the massive graviton can be given by

$$
\begin{aligned}
\phi & =\phi_{1} \cos [m t]+\phi_{2} \sin [m t], \\
B & =B_{1} \cos [m t]+B_{2} \sin [m t], \\
C & =C_{1} \cos [m t]+C_{2} \sin [m t], \\
\psi & =\psi_{1} \cos [m t]+\psi_{2} \sin [m t], \\
\delta \varphi & =\delta \varphi_{1} \cos [m t]+\delta \varphi_{2} \sin [m t],
\end{aligned}
$$

with slowly varying functions of time $\left\{\phi_{1,2}, B_{1,2}, C_{1,2}\right.$, $\left.\psi_{1,2}, \delta \varphi_{1,2}\right\}$. We shall show the particular solutions for the large scales (5.4) and the small scales (5.7) in order.

\section{A. Large scale inhomogeneity}

In the large scales (5.4), the consistency of the equations leads to the following amplitudes for the variables:

$$
\begin{aligned}
\Phi, \Psi, H_{T}, \delta \varphi_{2} & =\mathcal{O}\left(m^{0}\right), \\
B_{1}, C_{1}, \psi_{1}, \delta \varphi_{1} & =\mathcal{O}\left(m^{-1}\right), \\
\phi_{1,2}, B_{2}, C_{2}, \psi_{2} & =\mathcal{O}\left(m^{-2}\right) .
\end{aligned}
$$

The order $\mathcal{O}\left(\mathrm{m}^{-2}\right)$ quantities give just sub-subleading contributions thus we can ignore them.

Equations (5.11) and (5.12) yield the constraint equations which determine $B_{1}, C_{1}$ and $\psi_{1}$ as

$$
B_{1}=-\frac{4 k}{m a} \delta \varphi_{2}, \quad C_{1}=\frac{2 k}{m a} \delta \varphi_{2}, \quad \psi_{1}=\frac{2 k_{\|}^{4}}{k^{4}} H_{T} \bar{\varphi}_{1} .
$$

Equation (5.10) gives

$$
\delta \dot{\varphi}_{2}+\frac{3}{2} H \delta \varphi_{2}+m \Phi \bar{\varphi}_{1}=0 .
$$

We do not find other equations within our accuracy.

After using the above equation and taking the time average, we obtain

$$
\begin{gathered}
\delta \rho_{G}=6 m^{2}\left[\bar{\varphi}_{1} \delta \varphi_{1}+\bar{\varphi}_{2} \delta \varphi_{2}+\bar{\varphi}_{1}^{2}\left(2 \Psi+\frac{k_{\|}^{4}}{k^{4}} H_{T}\right)\right], \\
v_{S}=-\frac{k}{a m \bar{\varphi}_{1}} \delta \varphi_{2},
\end{gathered}
$$




$$
v_{V}, \delta p_{G}, \pi_{S}, \pi_{V}, \pi_{T}=0,
$$

from the perturbed graviton energy-momentum tensor. Therefore, the graviton energy-momentum tensor is given by a form of pressureless perfect fluid without the vectortype components (i.e., an irrotational fluid). Although the irrotational property is obtained because of our specific ansatz (5.31), the pressureless property is hold even if we consider the general solution.

Note that the evolution of $\delta \varphi_{1}$ (and also $\bar{\varphi}_{2}$ ) has not been determined within our accuracy. We can, however, determine the dynamics of $\delta \rho_{G}$ by choosing the combinations $\delta \rho_{G}$ as an independent variable instead of $\delta \varphi_{1}$. The dynamics of $\delta \rho_{G}$ is determined by the conservation law of the averaged graviton energy-momentum tensor ${ }^{6}$ which reads

$$
\begin{gathered}
\delta \dot{\rho}_{G}+3 H \delta \rho_{G}+\left(\frac{k}{a} v_{S}+3 \dot{\Psi}\right) \bar{\rho}_{G}=0, \\
\dot{v}_{S}+H v_{S}-\frac{k}{a} \Phi=0,
\end{gathered}
$$

where we notice that (5.40) is exactly same as Eq. (5.35). Equation (5.39) determines the dynamics of $\delta \rho_{G}$.

Although the definition of $\delta \rho_{G}$ contains the tensor mode $H_{T}$, Eq. (5.39) indicates that the dynamics of $\delta \rho_{G}$ is independent from the tensor mode $H_{T}$. Therefore, if we focus on only the macroscopic behaviour of the massive graviton condensate (i.e., we focus on only the dynamics of $\left\langle T_{G}^{\mu \nu}\right\rangle_{T}$ ), the scalar modes and the tensor mode are decoupled. Needless to say, at the microscopic level, the scalar modes and the tensor modes should be coupled. For example, the dynamics of $\delta \varphi_{1}$ would depend on the dynamics of the tensor mode $H_{T}$ as well as the scalar modes $\Phi, \Psi$. Furthermore, the couplings between the scalar-vector-tensor modes would appear when we consider more higher-order corrections. The present calculations are justified up to the sub-subleading order.

As a result, the massive graviton condensate behaves as a dust fluid in the large scales (5.4) even if a small inhomogeneity is introduced. The massive graviton condensate can cluster and then explain the cosmic structure formation.

\section{B. Small scale inhomogeneity}

Next, we discuss the solution in the small scales (5.7) in which the amplitudes are given by

\footnotetext{
${ }^{6} \mathrm{We}$ notice again that although the conservation law of the nonaveraged graviton energy-momentum tensor $\stackrel{(0)}{\nabla}_{\mu} T_{G}^{\mu \nu}=0$ is equivalent to the equation of motion of $\varphi_{\mu \nu}$, that of the averaged graviton energy-momentum tensor $\nabla_{\mu}^{(0)}\left\langle T_{G}^{\mu \nu}\right\rangle_{T}=0$ is not. Hence we can obtain the equation for $\delta \rho_{G}$ even if the equations for $\delta \varphi_{1}$ cannot be obtained from the equation of motion within our accuracy.
}

$$
\begin{aligned}
\Phi, \Psi, H_{T}, \delta \varphi_{1,2} & =\mathcal{O}\left(m^{0}\right), \\
B_{1,2}, C_{1,2} & =\mathcal{O}\left(m^{-1 / 2}\right), \\
\phi_{1,2}, \psi_{1,2} & =\mathcal{O}\left(m^{-1}\right) .
\end{aligned}
$$

The constraint equations (5.11) and (5.12) yield

$$
\begin{aligned}
\phi_{1}+3 \psi_{1}-6 \frac{k_{\|}^{4}}{k^{4}} H_{T} \bar{\varphi}_{1} & =0, \\
\phi_{2}+3 \psi_{2} & =0, \\
\pm \frac{2 m k}{a} \phi_{1,2}+\frac{k_{x}^{2}}{a^{2}} B_{2,1}+\frac{k_{\|}^{2}}{a^{2}} C_{2,1} & =0,
\end{aligned}
$$

and

$$
B_{1,2}=\mp \frac{4 k}{m a} \delta \varphi_{2,1}, \quad C_{1,2}= \pm \frac{2 k}{m a} \delta \varphi_{2,1},
$$

whereas (5.10) gives

$$
\begin{gathered}
\delta \dot{\varphi}_{1}+\frac{3}{2} H \delta \varphi_{1}-\frac{k^{2}}{2 m a^{2}} \delta \varphi_{2}=0, \\
\delta \dot{\varphi}_{2}+\frac{3}{2} H \delta \varphi_{2}+\frac{k^{2}}{2 m a^{2}} \delta \varphi_{1}+m \Phi \bar{\varphi}_{1}=0 .
\end{gathered}
$$

Note that there are ten independent equations for ten independent variables $\left\{\delta \varphi_{1,2}, B_{1,2}, C_{1,2}, \phi_{1,2}, \psi_{1,2}\right\}$. Hence, the dynamics of the massive graviton are completely determined within our accuracy, differently from the previous case.

We notice that the equations (5.44) and (5.45) yield the Schrödinger equation in the cosmological background. The "wavefunction" $u(t, \mathbf{x})$ is defined by the relation

$\bar{\varphi}(t)+\delta \varphi(t, \mathbf{x})=\frac{1}{2}\left[u(t, \mathbf{x}) e^{-i m t}+u^{*}(t, \mathbf{x}) e^{i m t}\right]$,

where $\delta \varphi(t, \mathbf{x})$ is the variable in the real space which is used only here. Then, we obtain

$$
i\left(\frac{\partial u}{\partial t}+\frac{3}{2} H u\right)=\left(-\frac{\partial^{2}}{2 m^{2} a^{2}}+m \Phi\right) u,
$$

where $m \Phi u \simeq m \Phi \bar{\varphi}_{1}$ since we have considered the linearized theory. The wavefunction $u$ is dominated by the coherent mode $u_{0}=\bar{\varphi}_{1}$ which suggests that the almost homogeneous configuration of $\varphi_{\mu \nu}$ represents the condensate of the massive graviton. A large fraction of massive gravitons occupies the state $u_{0}$ except for the tiny perturbations $\delta \varphi_{\mu \nu}$. Note that $u_{0}$ is also a solution to the Schrödinger equation since the gravitational potential $\Phi$ is zero for the homogeneous configuration. 
We obtain

$$
\begin{gathered}
\delta \rho_{G}=6 m^{2} \bar{\varphi}_{1} \delta \varphi_{1}+\mathcal{O}\left(m^{0}\right), \\
v_{S}=-\frac{k}{a m \bar{\varphi}_{1}} \delta \varphi_{2}, \\
\delta p_{G}=-\frac{\bar{\varphi}_{1}}{6 a^{2}}\left(11 k_{x}^{2}+5 k_{\|}^{2}\right) \delta \varphi_{1}, \\
\pi_{S}=-\frac{\bar{\varphi}_{1}}{2 a^{2}}\left(10 k_{x}^{2}+7 k_{\|}^{2}\right) \delta \varphi_{1}, \\
\pi_{T}=-\frac{3 \bar{\varphi}_{1}}{2 a^{2}} k^{2} \delta \varphi_{1},
\end{gathered}
$$

and

$$
v_{V}=\pi_{V}=0
$$

Therefore, the massive graviton condensate is no longer recognized as a pressureless fluid. The effect of the pressure is relevant within the Jeans length. The Jeans momentum $k_{J}$ is estimated by the relation

$$
\left|\frac{k^{2}}{a^{2}} \frac{\delta p_{G}}{\delta \rho_{G}}\right|_{k=k_{J}} \sim \frac{\bar{\rho}_{G}}{M_{\mathrm{pl}}^{2}},
$$

which yields

$$
\frac{k_{J}^{2}}{a^{2}} \sim m H
$$

where we have assumed $M_{\mathrm{pl}}^{2} H^{2} \sim \bar{\rho}_{G}$. Hence, (5.7) indeed correspond to the scales below the Jeans scale.

If the massive graviton condensate is the dominant component, the Einstein equation yield

$$
\frac{k^{2}}{a^{2}} \Psi=\frac{1}{2 M_{\mathrm{pl}}^{2}} \delta \rho_{G}, \quad \frac{k^{2}}{a^{2}}(\Phi+\Psi)=-\frac{\pi_{S}}{M_{\mathrm{pl}}^{2}} .
$$

Since $\pi_{S}$ is of order $\mathcal{O}\left(m^{0}\right)$, we obtain $\Psi=-\Phi+\mathcal{O}\left(m^{-1}\right)$. Equation (5.45) then becomes

$$
\delta \dot{\varphi}_{2}+\frac{3}{2} H \delta \varphi_{2}+\left(\frac{k^{2}}{2 m a^{2}}-\frac{m a^{2}}{k^{2}} \frac{\bar{\rho}_{G}}{M_{\mathrm{pl}}^{2}}\right) \delta \varphi_{1}=0 .
$$

Combining with Eq. (5.44), we find

$$
\ddot{\delta}+2 H \dot{\delta}+\left(\frac{k^{4}}{4 m^{2} a^{4}}-\frac{\bar{\rho}_{G}}{2 M_{\mathrm{pl}}^{2}}\right) \delta=0,
$$

where $\delta:=\delta \rho_{G} / \bar{\rho}_{G}=2 \delta \varphi_{1} / \bar{\varphi}_{1}$ is the relative perturbation of the energy density. Clearly, in the scales beyond the Jeans scale, this equation admits a growing mode solution $\delta \propto a$ due to the Jeans instability in the dust-dominant universe. On the other hand, we find

$$
\delta \propto \exp \left[ \pm i \frac{3 k^{2}}{2 m a^{2}} t\right]
$$

for $k^{2} \gg k_{J}^{2}$ with $a \propto t^{2 / 3}$. The massive graviton condensate shows the acoustic oscillation.

The tensor mode is not decoupled from the scalar mode. The gravitational wave $H_{T}$ is sourced by the anisotropic stress $\pi_{T}$ which is related with the energy density of the massive graviton condensate. The acoustic oscillation of the massive graviton emits the gravitational waves.

\section{PRODUCTION OF MASSIVE GRAVITON CONDENSATE}

As shown in the previous section, the massive graviton condensate is indeed a candidate of dark matter. We thus consider a production mechanism of the massive graviton condensate and discuss whether the massive graviton condensate can be the dominant component of dark matter.

To generate the massive graviton condensate we need an anisotropic source which is coherent on the cosmological scale. $^{7}$ A candidate is the cosmological scale magnetic field. The blazar observations suggests the lower bound of the strength of the extragalactic magnetic field $B_{0}$ is about $10^{-17} \mathrm{G}$. The upper bound is obtained from the CMB observations which is about $10^{-9} \mathrm{G}$ [47]. Since the production and the evolution of the cosmological scale magnetic field are subject to discussion (see [48-50] for reviews), we consider the simplest scenario.

We assume that the coherent magnetic field is generated in the early Universe, e.g., in the inflationary regime of the Universe. Here, we do not discuss the initial spectrum of the dark matter perturbations which should depend on the details of the coherent magnetic field. We just estimate the produced amount of the coherent massive gravitons. If the magnetic field adiabatically evolves, the energy density decreases as $a^{-4}$. On the other hand, the energy density of the massive graviton decreases as $a^{-3}$. Therefore, the production of the massive graviton condensate by the magnetic field can be ignored in the late stage of the Universe $(m \gg H)$.

The separation (3.7) is not justified in $H \gtrsim m$. To discuss the early Universe we have to directly analyze the Bianchi universe which is summarized in Appendix B. By solving Eqs. (B9)-(B13), a typical behavior of $\bar{\varphi}$ produced by the coherent magnetic field is shown in Fig. 1.

\footnotetext{
${ }^{7}$ Although we have assumed that the massive graviton is coherent on the cosmological scale for simplicity, we can discuss the case when the coherent scale is smaller than the horizon scale but larger than, at least, the de Broglie wavelength of the massive graviton. The source is not necessary to be anisotropic over the cosmological scale.
} 


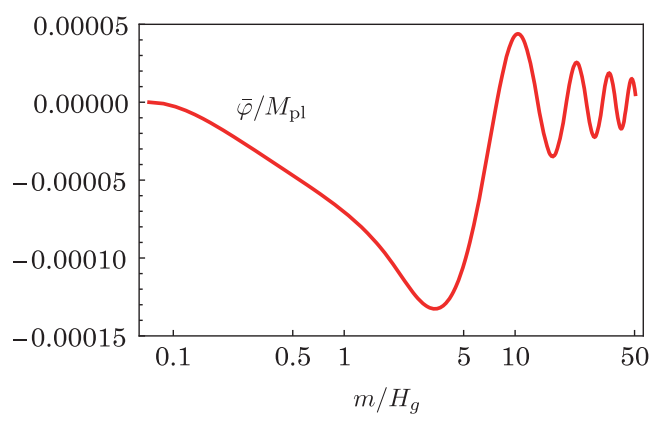

FIG. 1. A production of $\bar{\varphi}$ by the coherent magnetic field with $\Omega_{B} / \Omega_{r}=10^{-4}$. $H_{g}$ is the Hubble expansion rate of the $g$ spacetime which becomes $H_{g} \simeq H$ in $H_{g} \ll m$. We set $b_{1}=$ -1 and initially set both the $g$-spacetime and the $f$-spacetime are isotropic, i.e., $\bar{\varphi}=0$ and $\dot{\bar{\varphi}}=0$.

The amplitude of $\varphi_{\mu \nu}$ does not grow in $H \gg m$ since the Hubble friction is too large. As a result, the dominant production of the massive graviton condensate should occur at $H \sim m$. The produced amplitude of the condensate is estimated as

$$
\left|\bar{\varphi}_{*}\right| \sim \frac{B_{*}^{2}}{M_{\mathrm{pl}} m^{2}} \sim \frac{B_{*}^{2}}{M_{\mathrm{pl}} H_{*}^{2}} \sim M_{\mathrm{pl}} \frac{\Omega_{B}}{\Omega_{r}},
$$

where the asterisk represents the quantities at the production and $B$ is the strength of the magnetic field. $\Omega_{B}$ and $\Omega_{r}$ are the present density parameters of the coherent magnetic field and radiation, respectively. To obtain the final expression we use the Universe is dominated by radiation at the production, $M_{\mathrm{pl}}^{2} H_{*}^{2} \sim \rho_{r}^{*}$, and both $B^{2}$ and $\rho_{r}$ decrease as $a^{-4}$. Once the gravitons are produced, $\varphi_{\mu \nu}$ is decoupled from the magnetic field because the contribution from the interaction quickly decreases [see Eq. (B29)]. In order that $\varphi_{\mu \nu}$ is the dominant component of dark matter, the present amplitude has to be $\bar{\varphi}_{0} \sim M_{\mathrm{pl}} H_{0} / m$ where the present and the produced amplitudes are related by $a_{0}^{3 / 2} \bar{\varphi}_{0}=a_{*}^{3 / 2} \bar{\varphi}_{*}$. Then, we get the condition for the graviton mass being the dominant component of dark matter as

$$
m \sim H_{0} z_{\mathrm{eq}}^{-3 / 2}\left(\frac{\Omega_{r}}{\Omega_{B}}\right)^{4} \sim 10^{-4}\left(\frac{10^{-10} \mathrm{G}}{B_{0}}\right)^{8} \mathrm{eV} .
$$

where $z_{\text {eq }}$ is the redshift of the equality time. The graviton mass should be $10^{-4} \mathrm{eV} \lesssim m \lesssim 10^{7} \mathrm{eV}$ where the lower bound is obtained from laboratory-scale experiments of gravity while the upper bound is given by the lifetime of the massive graviton. Hence, a consistent scenario is constructed when the present magnetic field is

$$
10^{-12} \mathrm{G} \lesssim B_{0} \lesssim 10^{-10} \mathrm{G}
$$

which is indeed a viable region of the coherent magnetic field.

\section{SUMMARY AND DISCUSSIONS}

We provide a scenario in which a tiny deformation of the spacetime is dark matter in the ghost-free bigravity theory. This deformation is interpreted as the "condensate" of the massive gravitons. Differently from the case of the massless graviton, the zero momentum state of the massive graviton is well defined when $m^{2} \gg H^{2}$. We find that the zero momentum massive graviton with small fluctuations is a viable candidate of dark matter.

We have also studied a production mechanism of the coherent massive gravitons with the mass range $10^{-4} \mathrm{eV} \lesssim$ $m \lesssim 10^{7} \mathrm{eV}$ and shown the coherent magnetic field with $10^{-12} \mathrm{G} \lesssim B_{0} \lesssim 10^{-10} \mathrm{G}$ yields a sufficient amount of massive gravitons for dark matter. When the present value of the coherent magnetic field is determined by a future observation, we can fix a suitable value of the graviton mass to be dark matter.

Although we discussed the magnetic field as a source of the massive graviton condensate, another source to produce the condensate could exist. In general, if there exits a coherent anisotropic stress $\pi_{\text {coh }}$ whose coherent scale is $L \gtrsim$ $m^{-1}$ and the density is $\pi_{\mathrm{coh}} / \rho_{r} \sim 10^{-10}$ in the age $H \sim m$, the massive graviton condensate is produced and becomes dark matter. Since the gravitons universally couple to matter fields, the source is not necessary to be a standard model particle. Any matter field can be a source of the gravitons.

If the anisotropic stress is a random field instead of the coherent field, the stochastic massive gravitons are produced which have been discussed in [17]. Even for the stochastic case, we obtain a viable scenario of the massive graviton dark matter. Hence, if the anisotropic stress existed in the early Universe, it inevitably yields the stochastic or the coherent massive gravitons and then the massive gravitons can be dark matter.

One may interpret that the $Z_{2}$ symmetry is a fundamental symmetry of the massive graviton. Although we have assumed the $Z_{2}$ symmetry only for the self-interactions of gravitons, the symmetry can be introduced into the matter-graviton interactions as well. The $Z_{2}$ symmetry holds if the action is invariant under the replacement $g \leftrightarrow f$ in bigravity. At low energy scales, matter fields can couple to both metrics $g_{\mu \nu}$ and $f_{\mu \nu}$ via an effective composite metric [51-59]. Hence, when all matter fields couple to the composite metric $g_{\mu \nu}^{\text {eff }}$ defined by

$$
g_{\mu \nu}^{\text {eff }}=\frac{1}{4}\left[g_{\mu \nu}+2(\sqrt{g f})_{\mu \nu}+f_{\mu \nu}\right],
$$

with

$$
(\sqrt{g f})_{\mu \nu}=g_{\mu \alpha}\left(\sqrt{g^{-1} f}\right)_{\nu}^{\alpha},
$$

the matter-massive graviton interactions respect the $Z_{2}$ symmetry. Indeed, expanding the metrics under (3.7) we obtain 


$$
g_{\mu \nu}^{\mathrm{eff}}=\stackrel{(0)}{g}_{\mu \nu}+\frac{h_{\mu \nu}}{M_{\mathrm{pl}}}-\frac{1}{4 M_{\mathrm{pl}}^{2}} \varphi_{\mu \alpha} \varphi_{\nu}^{\alpha}+\cdots
$$

thus, the matter action is manifestly invariant under $\varphi_{\mu \nu} \rightarrow$ $-\varphi_{\mu \nu}$. In this case, the Yukawa interaction of the massive graviton does not appear and then the present graviton mass constraints cannot be applied. The details about the $Z_{2}$ symmetric bigravity theory are under investigation.

We have introduced the $Z_{2}$ symmetry to the selfinteractions of the massive graviton in order to simplify the calculations. However, the massive graviton condensate can be dark matter even without the $Z_{2}$ symmetry because the nonlinear terms are always sub-leading contributions and then may not affect the dynamics at leading order. The leading-order expression of $T_{G}^{\mu \nu}$ would be unchanged. In order that the massive graviton condensate is dark matter, the $Z_{2}$ symmetry of the self-interactions should not be required.

Our scenario can be directly confirmed when we observe the coherent anisotropic oscillation of the Universe. The frequency of the oscillation is unfortunately too high to detect the oscillation as a "gravitational wave" by the present and future gravitational wave detectors. However, if the graviton mass can be sufficently light due to, for example, the $Z_{2}$ symmetry, the coherent oscillation will be detectable.

Finally, we comment on an interesting remaining question: Is the almost homogeneous configuration of $\varphi_{\mu \nu}$ the Bose-Einstein condensate of the massive graviton? As is well known, a coherent massive scalar field, for example axion, is a viable dark matter candidate [60-74]. This coherent scalar field is interpreted as the Bose-Einstein condensate. Our result would be a generalization of the Bose-Einstein condensate of the massive scalar field to that of the massive tensor field. However, the present argument is completely classical and we have not discussed any quantum aspect of the massive graviton. Therefore, it would be interesting to study a connection to the quantum theory of the gravitation, but this is beyond the scope of the present paper.

\section{ACKNOWLEDGMENTS}

K. A. would like to thank Shinji Mukohyama and Ryo Namba for useful discussions and comments. This work was supported in part by Grants-in-Aid from the Scientific Research Fund of the Japan Society for the Promotion of Science (No. JP15J05540, No. JP16K05362, and No. JP17H06359).

\section{APPENDIX A: GRAVITON ENERGY- MOMENTUM TENSOR: CURVED BACKGROUND}

In this section, we summarize the definitions of the graviton energy-momentum tensors for generic cases. For completeness, we introduce both the $g$-matter fields $\psi_{g}$ and the $f$-matter fields $\psi_{f}$ and do not assume (3.32). The matter action is given by

$$
S^{[\mathrm{m}]}=S_{g}^{[\mathrm{m}]}\left(g, \psi_{g}\right)+\mathcal{S}_{f}^{[\mathrm{m}]}\left(f, \psi_{f}\right)
$$

and we denote the energy-momentum tensors of the $g$-matter field and the $f$-matter field as $T^{\mu \nu}$ and $\mathcal{T}^{\mu \nu}$, respectively.

To define the energy-momentum tensor of gravitons, we shall decompose the metric into the "background" and the "perturbations." However, in general, the deomposition into the perturbations and the background may not be well defined because the perturbations and the background interact with each others and then the equations may not be separable when the backreaction from the perturbations to the background is included. To decompose the Einstein equations, we assume the perturbations contain only highfrequency modes whereas the background consists of only the low-frequency modes. In this case, we obtain two independent equations for the low-frequency background and the high-frequency perturbations via a low-frequency projection $\langle\cdots\rangle_{\text {low }}$ and a high-frequency projection $\langle\cdots\rangle_{\text {high }}$, respectively. Therefore, we assume the metrics are expressed by the low-frequency backgrounds with the high-frequency perturbations:

$$
\begin{aligned}
& g_{\mu \nu}=g_{\mu \nu}^{(\text {low })}+\delta g_{\mu \nu}^{(\text {high }),} \\
& f_{\mu \nu}=f_{\mu \nu}^{(\text {low })}+\delta f_{\mu \nu}^{(\text {high }),}
\end{aligned}
$$

with $\left|\delta g_{\mu \nu}^{(\text {high })}\right| \ll\left|g_{\mu \nu}^{(\text {low })}\right|$ and $\left|\delta f_{\mu \nu}^{(\text {high })}\right| \ll\left|f_{\mu \nu}^{\text {(low) }}\right|$. The highfrequency mode and the low-frequency mode are defined by

$$
g_{\mu \nu}^{(\text {low })}=\left\langle g_{\mu \nu}\right\rangle_{\text {low }}, \quad \delta g_{\mu \nu}^{(\text {high })}=\left\langle g_{\mu \nu}\right\rangle_{\text {high }},
$$

which is same for $f_{\mu \nu}$. It is worth noting that we only assume the perturbations are high-frequency modes but do not assume the perturbations are high-momentum modes. In GR, the situations with the high-frequency waves and the situations with the high-momentum waves are equivalent since the graviton is massless. However, these situations are not equivalent in bigravity due to the existence of the massive graviton.

As already mentioned, the definitions of the massless mode and the massive mode are ambiguous in general. They can be defined when the curvature scale of the metrics is smaller than the graviton mass squared:

$$
\left|\partial_{\alpha} \partial_{\beta} g_{\mu \nu}\right| \ll m^{2}, \quad\left|\partial_{\alpha} \partial_{\beta} f_{\mu \nu}\right| \ll m^{2} .
$$

In this case, although the low-frequency modes of the massive gravitons can be excited by some source including matter as well as the backreactions from high-frequency modes, the amplitudes of the low-frequency massive modes must be tiny (Indeed, in Appendix B, we will see the amplitude of the low-frequency massive mode is suppressed by $\mathrm{m}^{-2}$ for the homogeneous matter distributions.). Hence, we may assume the background is 
approximated by the homothetic solution. Then, the spacetime are expressed as spacetimes are expressed as

$$
\begin{aligned}
& g_{\mu \nu}=\stackrel{(0)}{g}_{\mu \nu}+M_{\mu \nu}+\frac{h_{\mu \nu}}{M_{\mathrm{pl}}}+\frac{\varphi_{\mu \nu}}{M_{G}}, \\
& f_{\mu \nu}=\xi_{0}^{2}\left(\stackrel{(0)}{g}_{\mu \nu}-\alpha^{-1} M_{\mu \nu}+\frac{h_{\mu \nu}}{M_{\mathrm{pl}}}-\frac{\varphi_{\mu \nu}}{\alpha M_{G}}\right),
\end{aligned}
$$

where $\stackrel{(0)}{g}_{\mu \nu}$ and $M_{\mu \nu}$ are the low-frequency massless mode and the low-frequency massive mode while $h_{\mu \nu}$ and $\varphi_{\mu \nu}$ are the high-frequency massless mode and the low-frequency massive mode, respectively (see Table I). The assumption that the homothetic background is good approximation means $\left|M_{\mu \nu}\right| \ll\left|\stackrel{(0)}{g}_{\mu \nu}\right|$. In this case, $M_{\mu \nu}, h_{\mu \nu}$ and $\varphi_{\mu \nu}$ can be treated as the tensors with respect to $\stackrel{(0)}{g}_{\mu \nu}$.

The Ricci tensor for the $g$-spacetime is expanded as

$$
\begin{aligned}
R_{\mu \nu}= & \stackrel{(0)}{R}_{\mu \nu}+\delta \stackrel{(1)}{R}_{\mu \nu}[M]+\delta \stackrel{(1)}{R}_{\mu \nu}\left[\delta g^{(\text {high })}\right]+\delta \stackrel{(2)}{R}_{\mu \nu}[\Delta g] \\
& +\cdots,
\end{aligned}
$$

where $\Delta g_{\mu \nu}=M_{\mu \nu}+\delta g_{\mu \nu}^{(\text {high })}$. The high-frequency modes of $g_{\mu \nu}$ and $f_{\mu \nu}$ are written in terms of the mass eigenstates as

$$
\begin{aligned}
\delta g_{\mu \nu}^{(\text {high })} & =\frac{h_{\mu \nu}}{M_{\mathrm{pl}}}+\frac{\varphi_{\mu \nu}}{M_{G}}, \\
\delta f_{\mu \nu}^{(\text {high })} & =\xi_{0}^{2}\left(\frac{h_{\mu \nu}}{M_{\mathrm{pl}}}-\frac{\varphi_{\mu \nu}}{\alpha M_{G}}\right) .
\end{aligned}
$$

The functionals $\delta \stackrel{(1)}{R}_{\mu \nu}[\chi]$ and $\delta \stackrel{(2)}{R}_{\mu \nu}[\chi]$ are defined by

$$
\delta \stackrel{(1)}{R}_{\mu \nu}[\chi]=\frac{1}{2}\left(-\stackrel{(0)}{\nabla}_{\alpha} \stackrel{(0)}{\nabla}^{\alpha} \chi_{\mu \nu}-\stackrel{(0)}{\nabla}_{\mu} \stackrel{(0)}{\nabla}_{\nu} \chi_{\alpha}^{\alpha}+2 \stackrel{(0)}{\nabla}^{\alpha}{ }^{(0)}{ }_{(\mu} \chi_{\nu) \alpha}\right),
$$

$$
\begin{aligned}
& \delta \stackrel{(2)}{R}_{\mu \nu}[\chi]=\frac{1}{2} \stackrel{(0)}{g}_{\rho \sigma}{ }_{g}^{(0)} \alpha \beta\left[\frac{1}{2} \stackrel{(0)}{\nabla}_{\mu} \chi_{\alpha \rho} \stackrel{(0)}{\nabla}{ }_{\nu} \chi_{\sigma \beta}+2 \stackrel{(0)}{\nabla}_{\rho} \chi_{\nu \alpha} \stackrel{(0)}{\nabla}_{[\sigma} \chi_{\beta] \mu}\right. \\
& +\chi_{\rho \alpha}\left(\stackrel{(0)}{\nabla} \stackrel{(0)}{\nabla}_{\mu} \chi_{\sigma \beta}+\stackrel{(0)}{\nabla} \stackrel{(0)}{\beta}_{\sigma} \chi_{\mu \nu}-2 \stackrel{(0)}{\nabla} \stackrel{(0)}{\nabla}_{(\mu} \chi_{\nu) \sigma}\right) \\
& \left.+\left(\frac{1}{2} \stackrel{(0)}{\nabla}_{\alpha} \chi_{\rho \sigma}-\stackrel{(0)}{\nabla}_{\rho} \chi_{\alpha \sigma}\right)\left(2 \stackrel{(0)}{\nabla}_{\left(\mu \chi_{\nu}\right) \beta}-\stackrel{(0)}{\nabla}_{\beta} \chi_{\mu \nu}\right)\right] \text {, }
\end{aligned}
$$

for a tensor $\chi_{\mu \nu}$. The linear quantities in $M_{\mu \nu}$ and $\delta g_{\mu \nu}^{(\text {high })}$ are decomposed whereas the quadratic quantity $\delta \stackrel{(2)}{R}_{\mu \nu}[\Delta g]$ have the cross terms between $M_{\mu \nu}$ and $\delta g_{\mu \nu}^{(\text {high) }}$. The quadratic quantity is given by

$$
\begin{aligned}
\delta \stackrel{(2)}{R}_{\mu \nu}[\Delta g]= & \delta \stackrel{(2)}{R}_{\mu \nu}\left[\delta g^{(\text {high })}\right]+\delta \stackrel{(2)}{R}_{\mu \nu}[M] \\
& +\delta \stackrel{(2)}{R}_{\mu \nu}^{\operatorname{cross}}\left[M \times \delta g^{(\text {high })}\right],
\end{aligned}
$$

where the first two terms are quadratic in either $M_{\mu \nu}$ or $\delta g_{\mu \nu}^{\text {(high) }}$, respectively, and the third term represents the cross terms. Note that $\stackrel{(1)}{R}_{\mu \nu}[M]$ and $\stackrel{(2)}{R}_{\mu \nu}[M]$ are the purely low-frequency quantities while $\delta R_{\mu \nu}^{(1)}\left[\delta g^{(\text {high })}\right]$ and $\delta R_{\mu \nu}^{(2)}$ cross $\left[M \times \delta g^{(\text {high })}\right]$ are the purely high-frequency quantities with the inequalities

$$
\begin{aligned}
& \delta \stackrel{(1)}{R}_{\mu \nu}[M] \gg \stackrel{(2)}{R}_{\mu \nu}[M], \\
& \delta \stackrel{(1)}{R}_{\mu \nu}\left[\delta g^{(\text {high })}\right] \gg \stackrel{(2)}{R}_{\mu \nu}\left[M \times \delta g^{(\text {high })}\right] .
\end{aligned}
$$

After taking the high/low-frequency projections, we obtain

$$
\left\langle R_{\mu \nu}\right\rangle_{\text {low }}=\stackrel{(0)}{R}_{\mu \nu}+\stackrel{(1)}{R}_{\mu \nu}[M]+\left\langle\delta \stackrel{(2)}{R}_{\mu \nu}\left[\delta g^{(\text {high })}\right]\right\rangle_{\text {low }}+\cdots,
$$

$\left\langle R_{\mu \nu}\right\rangle_{\text {high }}=\delta \stackrel{(1)}{R}_{\mu \nu}\left[\delta g^{(\text {high })}\right]+\left\langle\delta \stackrel{(2)}{R}_{\mu \nu}\left[\delta g^{(\text {high })}\right]\right\rangle_{\text {high }}+\cdots$,

The other quantities are expanded in the similar way and then the equations are decomposed into ones for the low-frequency modes and for the high-frequency modes, respectively.

Up to the linear order the high-frequency mode equations are decomposed into the massless one and the massive one

$$
\begin{aligned}
& \delta \stackrel{(1)}{R}_{\mu \nu}[h]-\Lambda_{g} h_{\mu \nu}=\frac{1}{M_{\mathrm{pl}}}\left(\delta \stackrel{(1)}{S}_{\mu \nu}+\xi_{0}^{2} \delta \stackrel{(1)}{\mathcal{S}}_{\mu \nu}\right), \\
& \delta \stackrel{(1)}{R}_{\mu \nu}[\varphi]-\Lambda_{g} \varphi_{\mu \nu}+\frac{m_{\mathrm{eff}}^{2}}{4}\left(2 \varphi_{\mu \nu}+\varphi^{\alpha}{ }_{\alpha}^{(0)}{ }_{\mu \nu}\right) \\
& =\frac{1}{M_{G}}\left(\delta \stackrel{(1)}{S}_{\mu \nu}-\alpha^{-1} \xi_{0}^{2} \delta \stackrel{(1)}{\mathcal{S}}_{\mu \nu}\right),
\end{aligned}
$$

where we define

$$
\begin{aligned}
\delta \stackrel{(1)}{S}_{\mu \nu} & :=\left\langle T_{\mu \nu}-\frac{1}{2} g_{\mu \nu} T\right\rangle_{\text {high }}, \\
\delta \mathcal{S}_{\mu \nu}^{(1)} & :=\left\langle\mathcal{T}_{\mu \nu}-\frac{1}{2} f_{\mu \nu} \mathcal{T}\right\rangle_{\text {high }} .
\end{aligned}
$$

Note that $\left\langle\stackrel{(g)}{\nabla}{ }_{\mu} T_{(\text {int })}^{\mu \nu}\right\rangle_{\text {high }}=0$ leads to

$$
\stackrel{(0)}{\nabla}_{\mu} \varphi^{\mu}{ }_{\nu}=\stackrel{(0)}{\nabla}_{\nu} \varphi .
$$


Substituting this into the trace of (A17), we find a constraint equation on the trace $\varphi_{\alpha}^{\alpha}$

$$
\left(3 m_{\mathrm{eff}}^{2}-2 \Lambda_{g}\right) \varphi_{\alpha}^{\alpha}=\frac{2}{M_{G}}\left(\delta \stackrel{(1)}{S}_{\alpha \beta}-\alpha^{-1} \xi_{0}^{2} \delta \mathcal{S}_{\alpha \beta}\right)^{(1)}{ }^{\alpha \beta} .
$$

From the low-frequency mode equations, we obtain the Einstein equation for the homothetic background

$$
\stackrel{(0)}{G}^{\mu \nu}+\Lambda_{g} \stackrel{(0)}{g}^{\mu \nu}=\frac{1}{M_{\mathrm{pl}}^{2}} \tau^{\mu \nu},
$$

where $\stackrel{(0)}{G}^{\mu \nu}$ is the Einstein tensor for $\stackrel{(0)}{g}_{\mu \nu}$ and

$$
\tau^{\mu \nu}:=\stackrel{(0)}{T}^{\mu \nu}+\xi_{0}^{2} T^{\mu \nu}+\left\langle T_{\mathrm{gw}}^{\mu \nu}\right\rangle_{\mathrm{low}}+\left\langle T_{G}^{\mu \nu}\right\rangle_{\mathrm{low}},
$$

is interpreted as the total energy-momentum tensor including the gravitons as well as the matters. The effective matter energy-momentum tensors for matters $\stackrel{(0)}{T}_{\mu \nu}$ and $\stackrel{(0)}{\mathcal{T}}_{\mu \nu}$ are defined by the relations

$$
\stackrel{(0)}{S}_{\mu \nu}:=\left\langle T_{\mu \nu}-\frac{1}{2} g_{\mu \nu} T\right\rangle_{\text {low }}=\stackrel{(0)}{T}_{\mu \nu}-\frac{1}{2} \stackrel{(0)}{g}{ }_{\mu \nu}^{(0)} \underset{\alpha \beta}{\stackrel{(0)}{g}} \alpha \beta,
$$

$$
\stackrel{(0)}{\mathcal{S}}_{\mu \nu}:=\left\langle\mathcal{T}_{\mu \nu}-\frac{1}{2} f_{\mu \nu} \mathcal{T}\right\rangle_{\text {low }}=\stackrel{(0)}{\mathcal{T}}_{\mu \nu}-\frac{1}{2} \stackrel{(0)}{g}_{\mu \nu} \stackrel{(0)}{\mathcal{T}}_{\alpha \beta} \stackrel{(0)}{g}^{\alpha \beta},
$$

and the graviton energy-momentum tensors are defined by

$$
T_{\mathrm{gw}}^{\mu \nu}=-\left(\stackrel{(0)}{g} \mu \alpha \stackrel{(0)}{g} \nu \beta-\frac{1}{2} \stackrel{(0)}{g} \mu \stackrel{(0)}{g} \alpha \beta\right) \delta R_{\alpha \beta}^{(2)}[h],
$$

and (3.22).

Finally, we derive the equation for the massive mode $M_{\mu \nu}$ which is given by

$$
\delta \stackrel{(1)}{R}_{\mu \nu}[M]-\Lambda_{g} M_{\mu \nu}+\frac{m_{\mathrm{eff}}^{2}}{4}\left(2 M_{\mu \nu}+M_{\alpha}^{\alpha}{ }_{\alpha} \stackrel{(0)}{\mu}_{\mu \nu}\right)=\frac{\bar{\kappa}}{M_{G}} \Delta S_{\mu \nu},
$$

where

$$
\begin{aligned}
& \Delta S_{\mu \nu}:=\stackrel{(0)}{S}_{\mu \nu}-\alpha^{-1} \xi_{0}^{2} \stackrel{(0)}{\mathcal{S}}_{\mu \nu}+\left\langle-\frac{1}{\kappa_{g}^{2}} \delta \stackrel{(2)}{R}_{\mu \nu}\left[\delta g^{(\text {high })}\right]\right. \\
& \left.+\frac{1}{\xi_{0}^{4} \kappa_{g}^{2}} \delta \stackrel{(2)}{R}_{\mu \nu}\left[\delta f^{(\mathrm{high})}\right]+\Delta \stackrel{(2)}{S} \underset{\mu \nu}{(\mathrm{int})}\right\rangle_{\text {low }},
\end{aligned}
$$

and

$$
\begin{aligned}
\Delta \stackrel{(2)}{S}{ }_{\mu \nu}^{(i n t)}= & \frac{m_{\text {eff }}^{2}}{4 \alpha^{1 / 2}}\left(g_{\mu \nu} h^{\alpha \beta} \varphi_{\alpha \beta}-h_{\mu \nu} \varphi_{\alpha}^{\alpha}\right) \\
& -\frac{m_{\text {eff }}^{2}}{16 \alpha}\left[3(1-\alpha) \stackrel{(0)}{g}_{\mu \nu} \varphi_{\alpha \beta} \varphi^{\alpha \beta}\right. \\
& +4\left\{\left(1-\beta_{2}\right) \alpha-\beta_{2}\right\} \varphi_{\mu \nu} \varphi_{\alpha}^{\alpha}+2\left\{\left(1+2 \beta_{2}\right) \alpha\right. \\
& \left.\left.-\left(3-2 \beta_{2}\right) \alpha\right\} \varphi_{\mu}{ }^{\alpha} \varphi_{\nu \alpha}\right] .
\end{aligned}
$$

Note that the source term $\Delta S_{\mu \nu}$ is given by the difference between two matter energy-momentum tensors whereas the source term for the massless mode is given by the sum of energy-momentum tensors. The massive mode can give an anti-gravity since the positiveness of the source is not guaranteed even if all energy of the sources are positive definite.

\section{APPENDIX B: SPACETIME DEFORMATION AS DARK MATTER}

In this section, we study the axisymmetric Bainchi type I universe and obtain the same conclusion as the main text but from the different picture: we observe the spacetime anisotropy as dark matter in bigravity. In this section, we do not assume either the smallness of the Hubble parameter (3.3) or the $Z_{2}$ symmetry (3.32).

We consider the simplest homogenous but anisotropic universe in bigravity:

$d s_{g}^{2}=-N_{g}^{2} d t^{2}+a_{g}^{2}\left[e^{4 \beta_{g}} d x^{2}+e^{-2 \beta_{g}}\left(d y^{2}+d z^{2}\right)\right]$,

$d s_{f}^{2}=-N_{f}^{2} d t^{2}+a_{f}^{2}\left[e^{4 \beta_{f}} d x^{2}+e^{-2 \beta_{f}}\left(d y^{2}+d z^{2}\right)\right]$,

where $\left\{N_{g}, N_{f}, a_{g}, a_{f}, \beta_{g}, \beta_{f}\right\}$ are functions of the time $t$. The Hubble expansion rates and the shears are defined by

$$
\begin{aligned}
& H_{g}:=\frac{\dot{a}_{g}}{a_{g} N_{g}}, \quad H_{f}:=\frac{\dot{a}_{f}}{a_{f} N_{f}}, \\
& \sigma_{g}:=\frac{\dot{\beta}_{g}}{N_{g}}, \quad \sigma_{f}:=\frac{\dot{\beta}_{f}}{N_{f}} .
\end{aligned}
$$

Just for simplicity, we consider only the $g$-matter field whose energy-momentum tensor is given by

$$
T^{\mu}{ }_{\nu}=\operatorname{diag}\left[-\bar{\rho}(t), \bar{p}_{\perp}(t), \bar{p}_{\|}(t), \bar{p}_{\|}(t)\right],
$$

where the pressure is decomposed into the isotropic part $\bar{p}$ and the anisotropic part $\bar{\pi}$ :

$$
\begin{aligned}
& \bar{p}=\frac{1}{3}\left(\bar{p}_{\perp}+2 \bar{p}_{\|}\right), \\
& \bar{\pi}=\frac{1}{3}\left(\bar{p}_{\perp}-\bar{p}_{\|}\right) .
\end{aligned}
$$


The matter is assumed to satisfy the conservation law

$$
\dot{\bar{\rho}}+3 \frac{\dot{a}_{g}}{a_{g}}(\bar{\rho}+\bar{p})+6 \dot{\beta}_{g} \bar{\pi}=0 .
$$

Choosing the gauge $N_{g}=1$, we find following equations: the Friedmann equations

$$
\begin{aligned}
3 H_{g}^{2}= & \kappa_{g}^{2} \bar{\rho}+3 \sigma_{g}^{2}+m_{g}^{2}\left[b_{0}+b_{1}\left(e^{-2 \beta}+2 e^{\beta}\right) \xi\right. \\
& \left.+b_{2}\left(2 e^{-\beta}+e^{2 \beta}\right) \xi^{2}+b_{3} \xi^{3}\right], \\
3 H_{f}^{2}= & 3 \sigma_{f}^{2}+m_{f}^{2}\left[b_{4}+b_{3}\left(2 e^{-\beta}+e^{2 \beta}\right) \xi^{-1}\right. \\
& \left.+b_{2}\left(e^{-2 \beta}+2 e^{\beta}\right) \xi^{-2}+b_{1} \xi^{-3}\right],
\end{aligned}
$$

the constraint equation

$$
\begin{aligned}
H_{g} & {\left[3 b_{1}+2 b_{2} \xi\left(2 e^{\beta}+e^{-2 \beta}\right)+b_{3} \xi^{2}\left(e^{2 \beta}+2 e^{-\beta}\right)\right] } \\
& -H_{f} \xi\left[3 b_{3} \xi^{2}+2 b_{2} \xi\left(e^{2 \beta}+2 e^{-\beta}\right)+b_{1}\left(2 e^{\beta}+e^{-2 \beta}\right)\right] \\
& -2 \xi\left(e^{-\beta}-e^{2 \beta}\right)\left[\sigma_{f}\left(b_{1} e^{-\beta}+b_{2} \xi\right)+\sigma_{g}\left(b_{2} e^{-\beta}+b_{3} \xi\right)\right] \\
= & 0,
\end{aligned}
$$

and the equations for anisotropies

$$
\begin{aligned}
& \frac{1}{a_{g}^{3}} \frac{d}{d t}\left(a_{g}^{3} \sigma_{g}\right)+\kappa_{g}^{2} \frac{\partial U_{\beta}}{\partial \beta}=\kappa_{g}^{2} \bar{\pi} \\
& \frac{1}{a_{g}^{3}} \frac{d}{d t}\left(a_{f}^{3} \sigma_{f}\right)-\kappa_{f}^{2} \frac{\partial U_{\beta}}{\partial \beta}=0
\end{aligned}
$$

where we define

$$
\begin{gathered}
\xi:=\frac{a_{f}}{a_{g}}, \quad \beta:=\beta_{g}-\beta_{f}, \\
m_{g}^{2}:=m^{2} \frac{\kappa_{g}^{2}}{\kappa^{2}}, \quad m_{f}^{2}:=m^{2} \frac{\kappa_{f}^{2}}{\kappa^{2}},
\end{gathered}
$$

and

$$
\begin{aligned}
U_{\beta}:= & \frac{m^{2}}{6 \kappa^{2}}\left[\xi\left(2 e^{\beta}+e^{-2 \beta}\right)\left(b_{1}+b_{2} N_{f}\right)\right. \\
& \left.+\xi^{2}\left(e^{2 \beta}+2 e^{-\beta}\right)\left(b_{2}+b_{3} N_{f}\right)\right] .
\end{aligned}
$$

Equations (B12) and (B13) yield

$$
\frac{1}{a_{g}^{3}} \frac{d}{d t}\left[a_{g}^{3}\left(\kappa_{f}^{2} \sigma_{g}+\kappa_{g}^{2} \xi^{3} \sigma_{f}\right)\right]=\kappa_{g}^{2} \kappa_{f}^{2} \bar{\pi} .
$$

Hence, when the anisotropic stress is ignored $\bar{\pi}=0$, the sum of the shears $\sigma_{g}$ and $\sigma_{f}$ decreases as $a_{g}^{-3}$ which is the same as the standard decaying law of the shear in GR. On the other hand, the difference between them does not

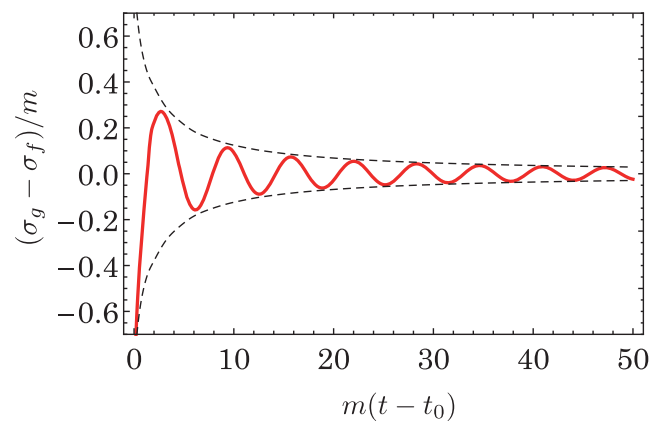

FIG. 2. The evolution of $\sigma_{g}-\sigma_{f}$ in vacuum $\bar{\rho}=\bar{p}=\bar{\pi}=0$ where the coupling constants are chosen as $\kappa_{g}^{2}=\kappa_{f}^{2}$ and (3.36) with $b_{2}=-1$. The initial values are $\beta\left(t_{0}\right)=0.1, \sigma_{g}\left(t_{0}\right)=-m$, $\sigma_{f}\left(t_{0}\right)=0.1 \mathrm{~m}$. The black dashed curves are proportional to $a_{g}^{-3 / 2}$.

decreases as $a_{g}^{-3}$ due to the "potential" $U_{\beta}$. Instead, $\sigma_{g}-\sigma_{f}$ (and also $\beta_{g}-\beta_{f}$ ) decrease as $a_{g}^{-3 / 2}$ as shown in Fig. 2 and then acts as the "dark matter" component of the Universe (see also [22]).

Figure 3 shows a typical behavior of the solution in vacuum. The top figure represents the spacetimes approach the homothetic solution and the bottom figure shows that $H_{g}^{2}$ decreases as $a_{g}^{-3}$ at the late time where we assume $\left.\Lambda_{g}\right|_{\xi_{0}=1}=0$. Therefore, even if the matter component is not introduced, the same behavior as the dust-dominant universe can be obtained.
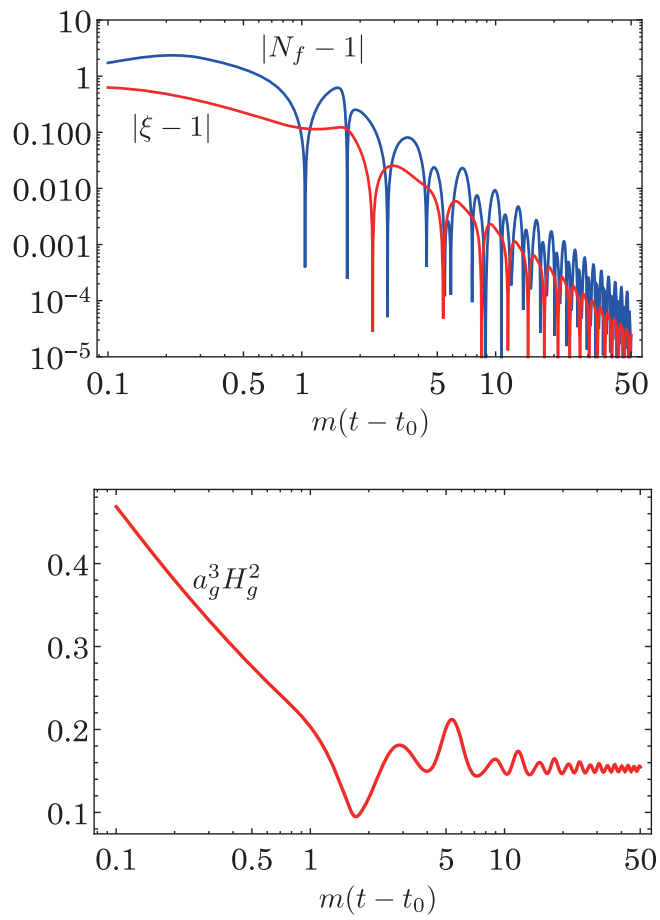

FIG. 3. The evolutions of $N_{f}, \xi$ and the rescaled Hubble expansion rate (which is scale free due to the scale factor). We set the same parameters as Fig. 2. 
We then assume $\beta \ll 1$ and consider the regime $m^{2} \gg H_{g}^{2}, H_{f}^{2}$. Note that the smallness of $\beta$ do not suggest that contributions of those to the Friedmann equations are also small because the quantity $m^{2} \beta^{2}$ can be comparable to $H_{g}^{2}$ and $H_{f}^{2}$. We introduce a small quantity $\epsilon:=H_{g} / m$ and set $\beta=\mathcal{O}(\epsilon)$. Equation (B11) gives

$$
\left(H_{g}-H_{f} \xi\right)\left(b_{1}+2 b_{2} \xi+b_{3} \xi^{2}\right)+\mathcal{O}\left(H_{g} \epsilon^{1}\right)=0,
$$

where we have used $\sigma_{g}, \sigma_{f} \lesssim H_{g}, H_{f}$. Since the spacetime can be approximated by the homothetic spacetime in the regime $m^{2} \gg H_{g}^{2}, H_{g}^{2}$, the ratio $\xi$ can be expanded around $\xi_{0}$. Supposing the normal branch such that

$$
H_{g}=\xi H_{f}+\mathcal{O}\left(H_{g} \epsilon\right),
$$

the Friedmann equations yield

$\left(3 m_{\mathrm{eff}}^{2}-2 \Lambda_{g}\right) \frac{\xi-\xi_{0}}{\xi_{0}} \approx-\kappa_{g}^{2}\left(\bar{\rho}+\bar{\rho}_{\sigma_{g}}\right)+\xi_{0}^{2} \kappa_{f}^{2} \bar{\rho}_{\sigma_{f}}$,

which indicates $\xi-\xi_{0}=\mathcal{O}\left(\epsilon^{2}\right)$ where

$$
\begin{aligned}
& \bar{\rho}_{\sigma_{g}}:=\frac{1}{\kappa_{g}^{2}}\left[3 \sigma_{g}^{2}+3 m_{g}^{2} \beta^{2}\left(b_{1} \xi_{0}+b_{2} \xi_{0}^{2}\right)\right], \\
& \bar{\rho}_{\sigma_{f}}:=\frac{1}{\kappa_{f}^{2}}\left[3 \sigma_{f}^{2}+3 m_{f}^{2} \beta^{2}\left(b_{2} \xi_{0}^{-1}+b_{3} \xi_{0}^{-2}\right)\right] .
\end{aligned}
$$

The deviation of the lapse function is then given by

$$
N_{f}-\xi_{0}=\frac{1}{H_{f}}\left(\frac{\dot{\xi}}{\xi}+H_{g}\right)-\xi_{0}=\mathcal{O}\left(\epsilon^{2}\right)
$$

where we notice that, although $\dot{\xi}$ and $H_{g}$ have quantities of order $\mathcal{O}(\epsilon)$, they are canceled and then $N_{f}-\xi_{0}$ is of order $\mathcal{O}\left(\epsilon^{2}\right)$. Using $m_{\text {eff }}^{2} \gg \Lambda_{g}$ and including only leading-order contributions, the Friedmann equation is expressed by

$$
3 H_{g}^{2} \approx \Lambda_{g}+\frac{1}{M_{\mathrm{pl}}^{2}}\left(\bar{\rho}+\bar{\rho}_{h}+\bar{\rho}_{G}\right)
$$

where

$$
\bar{\rho}_{h}:=3 \dot{\bar{h}}^{2}, \quad \bar{\rho}_{G}:=3\left(\dot{\bar{\varphi}}^{2}+m_{\mathrm{eff}}^{2} \bar{\varphi}^{2}\right),
$$

and

$$
\begin{aligned}
& \bar{\varphi}=\frac{1}{\bar{\kappa}}\left(\beta_{g}-\beta_{f}\right), \\
& \bar{h}=\frac{\kappa_{f}}{\xi_{0} \kappa_{g} \bar{\kappa}} \beta_{g}+\frac{\xi_{0} \kappa_{g}}{\kappa_{f} \bar{\kappa}} \beta_{f} .
\end{aligned}
$$

The variables $\bar{\varphi}$ and $\bar{h}$ are the normalized massive mode and the normalized massless mode of the anisotropies, or, following the notion of the main text, they can be interpreted as the "massive graviton condensate" and the "massless graviton condensate", respectively, which obey

$$
\begin{array}{r}
\ddot{\bar{h}}+3 H_{g} \dot{\bar{h}} \approx \frac{1}{M_{\mathrm{pl}}} \overline{\bar{\pi}}, \\
\ddot{\bar{\varphi}}+3 H_{g} \dot{\bar{\varphi}}+m_{\mathrm{eff}}^{2} \bar{\varphi} \approx \frac{1}{M_{G}} \bar{\pi} .
\end{array}
$$

It is worth noting that although we have assumed the inequalities $\beta \ll 1$ and $m^{2} \gg H_{g}^{2}, H_{g}^{2}$ we have not used the high-frequency and the low-frequency projections in the present calculations.

When we ignore the anisotropic stress, the (averaged) energy densities of $\bar{h}$ and $\bar{\varphi}$ decrease as

$$
\bar{\rho}_{h} \propto a_{g}^{-6}, \quad\left\langle\bar{\rho}_{G}\right\rangle_{T} \propto a_{g}^{-3} .
$$

The effect of the homogeneous mode of the massless graviton can be ignored in time.

The averaged differences $\left\langle\xi-\xi_{0}\right\rangle_{T}$ and $\left\langle N_{f}-\xi_{0}\right\rangle_{T}$ correspond to the low-frequency massive mode $M_{\mu \nu}$ which are given by

$$
\left\langle\xi-\xi_{0}\right\rangle_{T}, \quad\left\langle N_{f}-\xi_{0}\right\rangle_{T} \sim \epsilon^{2} \propto m^{-2} .
$$

As we expected, $M_{\mu \nu}$ is suppressed by $m^{-2}$ which is just a sub-subleading contribution.

Finally, we discuss the production of the anisotropies. We assume the matter field is composed of radiation and the coherent magnetic field:

$$
\bar{\rho}_{r} \propto a_{g}^{-4}, \quad \bar{p}_{r}=\frac{1}{3} \bar{\rho}_{r}, \quad \bar{\pi}_{r}=0,
$$

and

$\bar{\rho}_{B}=\frac{\bar{B}^{2}}{2 a_{g}^{4}} e^{4 \beta_{g}}, \quad \bar{p}_{B}=\frac{1}{3} \bar{\rho}_{B}, \quad \bar{\pi}_{B}=-\frac{2}{3} \bar{\rho}_{B}$,

where $\bar{B}$ is a constant and the strength of the magnetic field is given by $\bar{B} e^{2 \beta_{g}} / a_{g}^{2}$. A typical behavior of $\bar{\varphi}$ is shown in Fig. $1 .^{8}$ The dominant production occurs just after $H_{g}=m_{\text {eff }}$ in which Eq. (B29) can be barely used. The produced amplitude is then estimated as

$$
\bar{\varphi} \sim \frac{\bar{\pi}}{M_{G} m_{\mathrm{eff}}^{2}} .
$$

\footnotetext{
${ }^{8}$ Although the Higuchi instability [75-82] exists in subhorizon scales in $H_{g} \gtrsim m$, the unstable modes should not affect the dynamics of the homogeneous mode due to the cosmological Vainshtain mechanism [83-85]. Thus, we may discuss the dynamics of $\bar{\varphi}$ even in $H_{g} \gtrsim m$.
} 
In the main text, since we have assumed $M_{G}=M_{\mathrm{pl}}$ and used the normalization $m_{\text {eff }}=m$, we obtain (6.1).

\section{APPENDIX C: GENERAL PERTURBATIONS}

In this section, we summarize the calculations for the general perturbations around the homogeneous solutions (4.1) and (4.2). We use the specific choice of the coupling constants $\kappa_{g}^{2}=\kappa_{f}^{2}$ and (3.36) to simplify the calculations.

\section{Harmonics expansion}

The homogeneity of the background solution leads to that all variables can be transformed into the momentum space. Furthermore, due to the rotational symmetry in the $y-z$ space, the perturbations are decomposed into the even-parity perturbations and the odd-parity perturbations and there is no interaction between the even- and the oddparity perturbations at linear order. The even- and oddparity perturbations are parametrized as

$$
\left.\begin{array}{c}
-a C Y_{a} \\
2 a^{2} D Y_{x a} \\
a^{2}\left[2(\psi-\delta \varphi) \delta_{a b} Y_{S}+2 E Y_{a b}\right]
\end{array}\right),
$$

$$
\mathcal{Y}_{T i j}=k^{-2}\left(\begin{array}{cc}
0 & k_{\|}^{2} \mathcal{Y}_{x a} \\
* & -2 k_{x}^{2} \mathcal{Y}_{a b}
\end{array}\right),
$$

which satisfy (5.17) and (5.18). Using the three dimensional harmonics, any three dimensional vector (or tensor) is uniquely decomposed into the scalar and the vector (and the tensor) quantities. For example, the even-parity perturbations and the odd-parity perturbations of the graviton energy-momentum tensor are given by (5.25) and (5.26), respectively.

\section{Perturbations for massless mode}

The low-frequency mode $\stackrel{(0)}{g}_{\mu \nu}$ has the gauge symmetry by which we can obtain (5.23) and (5.24).

The general form of the even parity metric perturbations is given by

$$
\delta g_{\mu \nu}=\left(\begin{array}{cc}
-2 \Phi Y_{S} & -a B_{i} \\
* & a^{2}\left[2 \Psi Y_{S} \delta_{i j}+H_{i j}\right]
\end{array}\right),
$$

with

$$
B_{i}=\left(B_{g} Y_{x}, C_{g} Y_{a}\right)
$$

and

$$
H_{i j}=\left(\begin{array}{cc}
4 h Y_{S} & 2 D_{g} Y_{x b} \\
* & -2 h Y_{S} \delta_{a b}+2 E_{g} Y_{a b}
\end{array}\right)
$$

where we note

$$
H^{i}{ }_{i}=0 \text {, }
$$

and

$$
\partial_{i} B^{i}=\left(k_{x}^{2} B_{g}+k_{\|}^{2} C_{g}\right) k^{-1} Y_{S}
$$




$$
\begin{aligned}
& \partial_{i} H^{i x}=\left(2 k_{\|}^{2} D_{g}-4 k^{2} h_{g}\right) k^{-1} Y_{x}, \\
& \partial_{i} H^{i a}=\left(2 k_{x}^{2} D_{g}+k_{\|}^{2} E_{g}+2 k^{2} h_{g}\right) k^{-1} Y^{a} .
\end{aligned}
$$

Under the gauge transformation

$$
x^{\mu} \rightarrow x^{\mu}+\xi_{(\text {even })}^{\mu},
$$

with

$$
\xi^{\mu}=\left(\xi^{0} Y_{S}, \xi^{1} Y_{x}, \xi^{2} Y^{a}\right),
$$

the perturbations transform as

$$
\begin{aligned}
& \Phi_{g} \rightarrow \Phi_{g}+\dot{\xi}^{0}, \\
& \Psi_{g} \rightarrow \Psi_{g}+H \xi^{0}+\frac{1}{3} k \xi_{L}, \\
& h_{g} \rightarrow h_{g}+\frac{k_{x}^{2}}{3 k} \xi^{1}-\frac{k_{\|}^{2}}{6 k} \xi^{2}, \\
& B_{g} \rightarrow B_{g}-\frac{k}{a} \xi^{0}-a \dot{\xi}^{1}, \\
& C_{g} \rightarrow C_{g}-\frac{k}{a} \xi^{0}-a \dot{\xi}^{2}, \\
& D_{g} \rightarrow D_{g}-\frac{k}{2}\left(\xi^{1}+\xi^{2}\right), \\
& E_{g} \rightarrow E_{g}-k \xi^{2},
\end{aligned}
$$

and then

$$
\begin{aligned}
\partial_{i} B^{i} & \rightarrow \partial_{i} B^{i}-\left(\frac{k^{2}}{a} \xi^{0}+a k \dot{\xi}_{L}\right) Y_{S}, \\
\partial_{i} H^{i x} & \rightarrow \partial_{i} H^{i x}-\left(\xi^{1}+\frac{1}{3} \xi_{L}\right) k^{2} Y_{x}, \\
\partial_{i} H^{i a} & \rightarrow \partial_{i} H^{i a}-\left(\xi^{2}+\frac{1}{3} \xi_{L}\right) k^{2} Y^{a},
\end{aligned}
$$

where

$$
k^{2} \xi_{L}=k_{x}^{2} \xi^{1}+k_{\|}^{2} \xi^{2} .
$$

Therefore one can fix the gauge so that

$$
\partial_{i} B^{i}=0, \quad \partial_{i} H^{i j}=0,
$$

in which $B^{i}$ and $H^{i j}$ are given by

$$
B^{i}=B_{V} Y_{V}^{i}, \quad H^{i j}=2 H_{T} Y_{T}^{i j},
$$

where $B_{V}$ and $H_{T}$ are functions of time.

The odd parity metric perturbations are

$$
\delta g_{\mu \nu}^{(\text {odd })}=\left(\begin{array}{cc}
0 & -a \mathcal{B}_{i} \\
* & a^{2} \mathcal{H}_{i j}
\end{array}\right),
$$

with

and

$$
\mathcal{B}_{i}=\left(0, \mathcal{B}_{g} \mathcal{Y}_{a}\right)
$$

$$
\mathcal{H}_{i j}=\left(\begin{array}{cc}
0 & \mathcal{D}_{g} \mathcal{Y}_{x b} \\
* & 2 \mathcal{E}_{g} \mathcal{Y}_{a b}
\end{array}\right),
$$

where we notice

$$
\begin{aligned}
\partial_{i} \mathcal{B}^{i} & =0, \\
\partial_{i} \mathcal{H}^{i x} & =0, \\
\partial_{i} \mathcal{H}^{i a} & =\left(k_{x}^{2} \mathcal{D}_{g}+k_{\|}^{2} \mathcal{E}_{g}\right) k^{-1} \mathcal{Y}^{a}, \\
\mathcal{H}^{i} & =0 .
\end{aligned}
$$

Under the gauge transformation

$$
x^{\mu} \rightarrow x^{\mu}+\xi_{(\text {odd })}^{\mu},
$$

with

$$
\xi_{\text {(odd) }}^{\mu}=\left(0,0, \xi_{\text {odd }} \mathcal{Y}^{a}\right),
$$

the metric perturbations transform as

$$
\begin{aligned}
\mathcal{B}_{g} & \rightarrow \mathcal{B}_{g}-a \dot{\xi}_{(\text {odd })} \\
\mathcal{D}_{g} & \rightarrow \mathcal{D}_{g}-k \xi_{(\text {odd })} \\
\mathcal{E}_{g} & \rightarrow \mathcal{E}_{g}-k \xi_{(\text {odd })}
\end{aligned}
$$

and then

$$
\partial_{i} \mathcal{H}^{i a} \rightarrow \partial_{i} \mathcal{H}^{i a}-k^{2} \xi_{(\text {odd })} \mathcal{Y}^{a} .
$$

One can choose the gauge

$$
\partial_{i} \mathcal{H}^{i a}=0
$$

in which $\mathcal{B}_{g}^{i}$ and $\mathcal{H}_{g}^{i j}$ corresponds to the vector perturbation and the tensor perturbation, respectively:

$$
\mathcal{B}^{i}=\mathcal{B}_{V} \mathcal{Y}_{V}^{i}, \quad \mathcal{H}_{i j}=2 \mathcal{H}_{T} \mathcal{Y}_{T}^{i j} .
$$

\section{Adiabatic expansion}

In addition to the linearization of the inhomogeneities, we shall take the adiabatic expansion in terms of $m^{-1}$. To verify the linearization the amplitudes of the inhomogeneities are of $O\left(m^{n}\right)$ with $n \leq 0$.

The equation (5.10) yields that, up to the subleading order, the massive graviton can be expressed as

$$
\delta \varphi_{\mu \nu}=\delta \varphi_{1 \mu \nu} \cos [m t]+\delta \varphi_{2 \mu \nu} \sin [m t],
$$

where $\delta \varphi_{1 \mu \nu}$ and $\delta \varphi_{2 \mu \nu}$ are slowly varying function in time ${ }^{9}$ We use the suffixes 1 and 2 to represent the slowly varying

\footnotetext{
${ }^{9}$ If we do not assume the $Z_{2}$ symmetry for the self-interactions, the frequency at the subleading order can differ from $m / 2 \pi$.
} 
functions in front of $\cos [m t]$ and $\sin [m t]$ [see (5.32)]. The orders of $\delta \varphi_{1 \mu \nu}$ and $\delta \varphi_{2 \mu \nu}$ are determined to be consistent with the equations.

\section{Odd parity}

We first study the odd-parity perturbations in which (5.12) is trivially satisfied. We discuss the large scales (5.4) and the small scales (5.7) in order.

\section{a. Large scales}

The consistency of the equations yield

$$
\mathcal{B}_{1,2}=\mathcal{O}\left(m^{-1}\right)
$$

and other variables are of order $\mathcal{O}\left(m^{0}\right)$.

Form the equations of motion (5.10) and (5.11) we obtain the constraint equations

$$
\begin{aligned}
\frac{m k}{a} \mathcal{B}_{2}+\frac{k_{x}^{2}}{a^{2}} \mathcal{D}_{1}+\frac{k_{\|}^{2}}{a^{2}} \mathcal{E}_{1} & =0, \\
\frac{m k}{a}\left(\mathcal{B}_{1}+2 \bar{\varphi}_{1} \mathcal{B}_{V}\right)-\frac{k_{x}^{2}}{a^{2}} \mathcal{D}_{2}-\frac{k_{\|}^{2}}{a^{2}} \mathcal{E}_{2} & =0,
\end{aligned}
$$

and the dynamical equations

$$
\begin{aligned}
\dot{\mathcal{D}}_{1,2}+\frac{3}{2} H \mathcal{D}_{1,2} & =0, \\
\dot{\mathcal{E}}_{1,2}+\frac{3}{2} H \mathcal{E}_{1,2} & =0,
\end{aligned}
$$

which leads to

$$
\mathcal{D}_{1,2}, \mathcal{E}_{1,2} \propto a^{-3 / 2} .
$$

Then, we obtain the velocity and the pressure of the massive graviton condensate as

$$
v_{\text {(odd) }}=\mathcal{B}_{V}-\frac{1}{2 m \bar{\varphi}_{1}} \frac{k_{x}^{2}}{a k} \mathcal{D}_{2}
$$

and

$$
\pi_{V}^{\text {(odd) }}=\pi_{T}^{\text {(odd) }}=\mathcal{O}\left(m^{-1}\right),
$$

which indicates that the massive graviton can be interpreted as the pressureless ideal fluid.

\section{b. Small scales}

From the consistency, we find

$$
\mathcal{B}_{1,2}=\mathcal{O}\left(m^{-1 / 2}\right), \quad \mathcal{B}_{V}=\mathcal{O}\left(m^{-1 / 2}\right),
$$

and others are of order $\mathcal{O}\left(m^{0}\right)$.
The constraint equations are given by

$$
\frac{m k}{a} \mathcal{B}_{1,2} \mp \frac{k_{x}^{2}}{a^{2}} \mathcal{D}_{2,1} \mp \frac{k_{\|}^{2}}{a^{2}} \mathcal{E}_{2,1}=0,
$$

while the dynamical equation are

$$
\begin{gathered}
\dot{\mathcal{D}}_{1,2}+\frac{3}{2} H \mathcal{D}_{1,2} \mp \frac{k^{2}}{2 m a^{2}} \mathcal{D}_{2,1}=0, \\
\dot{\mathcal{E}}_{1,2}+\frac{3}{2} H \mathcal{E}_{1,2} \mp \frac{k^{2}}{2 m a^{2}} \mathcal{E}_{2,1}=0 .
\end{gathered}
$$

The solutions are

$$
\mathcal{D}_{1,2}, \mathcal{E}_{1,2} \propto a^{-3 / 2} \exp \left[ \pm i \frac{3 k^{2}}{2 m a^{2}} t\right],
$$

when $a \propto t^{2 / 3}$ (the dust-dominant universe).

We then obtain the velocity

$$
v_{\text {(odd) }}=-\frac{1}{2 m \bar{\varphi}_{1}} \frac{k_{x}^{2}}{a k} \mathcal{D}_{2},
$$

and the anisotropic pressures

$$
\begin{aligned}
& \pi_{V}^{\text {(odd) }}=-\frac{3 k_{x}^{2}}{2 a^{2}} \bar{\varphi}_{1} \mathcal{D}_{1}, \\
& \pi_{T}^{\text {(odd) }}=-\frac{k^{2}}{4 a^{2}} \bar{\varphi}_{1}\left(\mathcal{D}_{1}+2 \mathcal{E}_{1}\right) .
\end{aligned}
$$

Since $\mathcal{D}_{1,2}$ and $\mathcal{E}_{1,2}$ are independent, we can choose $\mathcal{D}_{1,2}$ and $\mathcal{D}_{1,2}+2 \mathcal{E}_{1,2}$ as new variables which obey the same equation as (C48) and (C49). The vector quantities $v_{\text {(odd) }}$ and $\pi_{V}^{\text {(odd) }}$ evolve independently from the tensor quantity $\pi_{T}^{\text {(odd) }}$. As a result, the vector and the tensor modes are decoupled.

\section{Even parity}

\section{a. Large scales}

In the large scales (5.4), we obtain

$$
\begin{aligned}
\phi_{1,2}, \psi_{2} & =\mathcal{O}\left(m^{-2}\right), \\
B_{1,2}, \psi_{1}, \delta \varphi_{1} & =\mathcal{O}\left(m^{-1}\right),
\end{aligned}
$$

and others are of order $\mathcal{O}\left(m^{0}\right)$. Since the quantities of order $\mathcal{O}\left(\mathrm{m}^{-2}\right)$ are sub-subleading order contributions, $\phi_{1,2}$ and $\psi_{2}$ are irrelevant to the dynamics.

From (5.11) and (5.12), we find five constraint equations

$$
2 k_{\|}^{4} H_{T} \bar{\varphi}_{1}-k^{4} \psi_{1}=0,
$$

$$
\frac{m k}{a} B_{2}+\frac{2 k_{\|}^{2}}{a^{2}} D_{1}=0,
$$

$$
\frac{m k}{a} C_{2}+\frac{2 k_{x}^{2}}{a^{2}} D_{1}+\frac{k_{\|}^{2}}{a^{2}} E_{1}=0,
$$




$$
\begin{gathered}
\frac{m k}{a} B_{1}-\frac{4 m k_{\|}^{2}}{a k} B_{T} \bar{\varphi}_{1}-\frac{2 k_{\|}^{2}}{a^{2}} D_{2}+4 \frac{k^{2}}{a^{2}} \delta \varphi_{2}=0, \quad \text { (C58) } \\
-\frac{m k}{a} C_{1}+\frac{2 m k_{x}^{2}}{a k} B_{T} \bar{\varphi}_{1}+\frac{2 k_{x}^{2}}{k^{2}} D_{2}+\frac{k_{\|}^{2}}{a^{2}} E_{2}+\frac{2 k^{2}}{a^{2}} \delta \varphi_{2}=0,
\end{gathered}
$$

which determines $\left\{\psi_{1}, B_{1,2}, C_{1,2}\right\}$ in terms of other variables. The nontrivial components (5.10) yield

$$
\delta \dot{\varphi}_{2}+\frac{3}{2} H \varphi_{2}+m \Phi \bar{\varphi}_{1}=0
$$

and

$$
\begin{gathered}
\dot{D}_{1,2}+\frac{3}{2} H D_{1,2}=0, \\
\dot{E}_{1,2}+\frac{3}{2} H E_{1,2}=0 .
\end{gathered}
$$

We cannot find other equations within our accuracy.

We note that the amplitudes of $D_{1,2}$ and $E_{1,2}$ always decreases since the gravitational potential $\Phi$ does not affect the dynamics of $D_{1,2}$ and $E_{1,2}$. The Jeans instability does not lead to the growth of $D_{1,2}$ and $E_{1,2}$.

After taking the oscillation average, we find that the pressures are zero. The energy density and $v_{S}$ are given by (5.36) and (5.37), respectively. The vector mode of the velocity is

$$
v_{V}=B_{V}+\frac{k}{a m \bar{\varphi}_{1}} D_{2}
$$

The massive graviton condensate is a form of a pressureless perfect fluid. Clearly from the definitions of $\delta \rho_{G}, v_{S}$ and $v_{V}$, the vector mode is decoupled from other modes.

As already mentioned in the main text, the evolution of $\delta \varphi_{1}$ is not determined. However, the dynamics of $\delta \rho_{G}$ is determined by the conservation law of the averaged graviton energy-momentum tensor.

\section{b. Small scales}

Finally, we study the even-parity perturbations in the small scales (5.7). The consistency leads to

$$
\begin{aligned}
B_{V} & =\mathcal{O}\left(m^{-1 / 2}\right), \\
B_{1,2}, C_{1,2} & =\mathcal{O}\left(m^{-1 / 2}\right), \\
\phi_{1,2}, \psi_{1,2} & =\mathcal{O}\left(m^{-1}\right),
\end{aligned}
$$

and others are of order $\mathcal{O}\left(m^{0}\right)$.

We obtain the eight constraint equations in terms of the eight variables $\left\{\psi_{1,2}, \phi_{1,2}, B_{1,2}, C_{1,2}\right\}$ as follows:

$$
\begin{array}{r}
\phi_{1}+3 \psi_{1}-6 \frac{k_{\|}^{4}}{k^{4}} H_{T} \bar{\varphi}_{1}=0, \\
\phi_{2}+3 \psi_{2}=0 \\
\pm \frac{2 m k}{a} \phi_{1,2}+\frac{k_{x}^{2}}{a^{2}} B_{2,1}+\frac{k_{\|}^{2}}{a^{2}} C_{2,1}=0 \\
\pm \frac{m k}{a} B_{1,2}+2 \frac{k_{\|}^{2}}{a^{2}} D_{2,1}-4 \frac{k^{2}}{a^{2}} \delta \varphi_{2,1}=0 \\
\pm \frac{m k}{a} C_{2,1}+2 \frac{k_{x}^{2}}{a^{2}} D_{1,2}+\frac{k_{\|}^{2}}{a^{2}} E_{1,2}+2 \frac{k^{2}}{a^{2}} \delta \varphi_{1,2}=0
\end{array}
$$

Equation (5.10) gives six equations

$$
\begin{aligned}
\delta \dot{\varphi}_{1}+\frac{3}{2} H \delta \varphi_{1}-\frac{k^{2}}{2 m a^{2}} \delta \varphi_{2} & =0, \\
\delta \dot{\varphi}_{2}+\frac{3}{2} H \delta \varphi_{2}+\frac{k^{2}}{2 m a^{2}} \delta \varphi_{1}+m \Phi \varphi_{1} & =0, \\
\dot{D}_{1,2}+\frac{3}{2} H D_{1,2} \mp \frac{k^{2}}{2 m a^{2}} D_{2,1} & =0, \\
\dot{E}_{1,2}+\frac{3}{2} H E_{1,2} \mp \frac{k^{2}}{2 m a^{2}} E_{2,1} & =0,
\end{aligned}
$$

which determine the dynamics of $\left\{\delta \varphi_{1,2}, D_{1,2}, E_{1,2}\right\}$.

The energy density and the velocity are expressed as (5.48), (5.49) and

$$
v_{V}=\frac{k}{a m \bar{\varphi}_{1}} D_{2}
$$

The pressures are not zero and given by

$$
\delta p_{G}=-\frac{\bar{\varphi}_{1}}{6 a^{2} k^{2}}\left[k^{2}\left(11 k_{x}^{2}+5 k_{\|}^{2}\right) \delta \varphi_{1}-2 k_{x}^{2} k_{\|}^{2} D_{1}+k_{\|}^{4} E_{1}\right],
$$

$$
\pi_{S}=-\frac{\bar{\varphi}_{1}}{4 a^{2} k^{2}}\left[2 k^{2}\left(10 k_{x}^{2}+7 k_{\|}^{2}\right) \delta \varphi_{1}-2 k_{x}^{2} k_{\|}^{2} D_{1}+k_{\|}^{4} E_{1}\right],
$$

$$
\begin{aligned}
& \pi_{V}=3 \frac{k^{2}}{a^{2}} \bar{\varphi}_{1} D_{1}, \\
& \pi_{T}=-\frac{\bar{\varphi}_{1}}{4 a^{2}}\left[6 k^{2} \delta \varphi_{1}+2 k_{x}^{2} D_{1}+\left(2 k_{x}^{2}+k_{\|}^{2}\right) E_{1}\right],
\end{aligned}
$$

Since the vector-type perturbations are determined by $D_{1}$ and $D_{2}$ only, the vector perturbations are decoupled from other perturbations. However, the scalar-type perturbations and the tensor-type perturbations are affected by $\delta \varphi_{1}, D_{1}$ and $E_{1}$ and then they are not decoupled. 
[1] B. P. Abbott et al. (VIRGO, LIGO ScIENTIFIC collaborations), Observation of Gravitational Waves from a Binary Black Hole Merger, Phys. Rev. Lett. 116, 061102 (2016).

[2] B. P. Abbott et al. (VIRGO, LIGO ScIENTIFIC collaborations), Binary Black Hole Mergers in the First Advanced LIGO Observing Run, Phys. Rev. X 6, 041015 (2016).

[3] R. A. Isaacson, Gravitational radiation in the limit of high frequency. I. The linear approximation and geometrical optics, Phys. Rev. 166, 1263 (1968).

[4] R. A. Isaacson, Gravitational radiation in the limit of high frequency. II. Nonlinear terms and the effective stress tensor, Phys. Rev. 166, 1272 (1968).

[5] K. Hinterbichler, Theoretical aspects of massive gravity, Rev. Mod. Phys. 84, 671 (2012).

[6] C. de Rham, Massive gravity, Living Rev. Relativity 17, 7 (2014).

[7] A. Schmidt-May and M. von Strauss, Recent developments in bimetric theory, J. Phys. A 49, 183001 (2016).

[8] C. M. Will, The confrontation between general relativity and experiment, Living Rev. Relativity 17, 4 (2014).

[9] J. Murata and S. Tanaka, A review of short-range gravity experiments in the LHC era, Classical Quantum Gravity 32, 033001 (2015).

[10] C. de Rham, J. T. Deskins, A. J. Tolley, and S.-Y. Zhou, Graviton mass bounds, Rev. Mod. Phys. 89, 025004 (2017).

[11] M. Fierz and W. Pauli, On relativistic wave equations for particles of arbitrary spin in an electromagnetic field, Proc. R. Soc. A 173, 211 (1939).

[12] D. G. Boulware and S. Deser, Can gravitation have a finite range?, Phys. Rev. D 6, 3368 (1972).

[13] C. de Rham and G. Gabadadze, Generalization of the FierzPauli action, Phys. Rev. D 82, 044020 (2010).

[14] C. de Rham, G. Gabadadze, and A. J. Tolley, Resummation of Massive Gravity, Phys. Rev. Lett. 106, 231101 (2011).

[15] S. F. Hassan and R. A. Rosen, Bimetric gravity from ghostfree massive gravity, J. High Energy Phys. 02 (2012) 126.

[16] K. Hinterbichler and R. A. Rosen, Interacting spin-2 fields, J. High Energy Phys. 07 (2012) 047.

[17] K. Aoki and S. Mukohyama, Massive gravitons as dark matter and gravitational waves, Phys. Rev. D 94, 024001 (2016).

[18] M. S. Volkov, Cosmological solutions with massive gravitons in the bigravity theory, J. High Energy Phys. 01 (2012) 035 .

[19] M. von Strauss, A. Schmidt-May, J. Enander, E. Mortsell, and S. F. Hassan, Cosmological solutions in bimetric gravity and their observational tests, J. Cosmol. Astropart. Phys. 03 (2012) 042.

[20] M. Berg, I. Buchberger, J. Enander, E. Mortsell, and S. Sjors, Growth histories in bimetric massive gravity, J. Cosmol. Astropart. Phys. 12 (2012) 021.

[21] D. Comelli, M. Crisostomi, F. Nesti, and L. Pilo, FRW cosmology in ghost free massive gravity, J. High Energy Phys. 03 (2012) 067.

[22] K.-i. Maeda and M.S. Volkov, Anisotropic universes in the ghost-free bigravity, Phys. Rev. D 87, 104009 (2013).

[23] Y. Akrami, T. S. Koivisto, and M. Sandstad, Accelerated expansion from ghost-free bigravity: A statistical analysis with improved generality, J. High Energy Phys. 03 (2013) 099.
[24] Y. Akrami, T. S. Koivisto, D. F. Mota, and M. Sandstad, Bimetric gravity doubly coupled to matter: theory and cosmological implications, J. Cosmol. Astropart. Phys. 10 (2013) 046.

[25] K. Aoki and K.-i. Maeda, Cosmology in ghost-free bigravity theory with twin matter fluids: The origin of dark matter, Phys. Rev. D 89, 064051 (2014).

[26] K. Aoki and K.-i. Maeda, Dark matter in ghost-free bigravity theory: From a galaxy scale to the universe, Phys. Rev. D 90, 124089 (2014).

[27] E. Babichev, L. Marzola, M. Raidal, A. Schmidt-May, F. Urban, H. Veermäe, and M. von Strauss, Bigravitational origin of dark matter, Phys. Rev. D 94, 084055 (2016).

[28] E. Babichev, L. Marzola, M. Raidal, A. Schmidt-May, F. Urban, H. Veermäe, and M. von Strauss, Heavy spin-2 dark matter, J. Cosmol. Astropart. Phys. 09 (2016) 016.

[29] T. Vachaspati, Magnetic fields from cosmological phase transitions, Phys. Lett. B 265, 258 (1991).

[30] B. Ratra, Cosmological 'seed' magnetic field from inflation, Astrophys. J. 391, L1 (1992).

[31] W. D. Garretson, G. B. Field, and S. M. Carroll, Primordial magnetic fields from pseudoGoldstone bosons, Phys. Rev. D 46, 5346 (1992).

[32] G. Sigl, A. V. Olinto, and K. Jedamzik, Primordial magnetic fields from cosmological first order phase transitions, Phys. Rev. D 55, 4582 (1997).

[33] A. Neronov and I. Vovk, Evidence for strong extragalactic magnetic fields from Fermi observations of $\mathrm{TeV}$ blazars, Science 328, 73 (2010).

[34] F. Tavecchio, G. Ghisellini, L. Foschini, G. Bonnoli, G. Ghirlanda, and P. Coppi, The intergalactic magnetic field constrained by Fermi/LAT observations of the TeV blazar 1ES $0229+200$, Mon. Not. R. Astron. Soc. 406, L70 (2010).

[35] K. Dolag, M. Kachelriess, S. Ostapchenko, and R. Tomas, Lower limit on the strength and filling factor of extragalactic magnetic fields, Astrophys. J. 727, L4 (2011).

[36] W. Essey, S. Ando, and A. Kusenko, Determination of intergalactic magnetic fields from gamma ray data, Astropart. Phys. 35, 135 (2011).

[37] A. M. Taylor, I. Vovk, and A. Neronov, Extragalactic magnetic fields constraints from simultaneous $\mathrm{GeV}-\mathrm{TeV}$ observations of blazars, Astron. Astrophys. 529, A144 (2011).

[38] K. Takahashi, M. Mori, K. Ichiki, S. Inoue, and H. Takami, Lower bounds on magnetic fields in intergalactic voids from long-term GeV-TeV light curves of the blazar Mrk 421, Astrophys. J. 771, L42 (2013).

[39] W. Chen, J. H. Buckley, and F. Ferrer, Search for GeV $\gamma$-Ray Pair Halos Around Low Redshift Blazars, Phys. Rev. Lett. 115, 211103 (2015).

[40] T. Fujita and R. Namba, Pre-reheating magnetogenesis in the kinetic coupling model, Phys. Rev. D 94, 043523 (2016).

[41] P. Adshead, J. T. Giblin, T. R. Scully, and E. I. Sfakianakis, Magnetogenesis from axion inflation, J. Cosmol. Astropart. Phys. 10 (2016) 039.

[42] S. F. Hassan, A. Schmidt-May, and M. von Strauss, On consistent theories of massive spin-2 fields coupled to gravity, J. High Energy Phys. 05 (2013) 086. 
[43] J. A. R. Cembranos, A. L. Maroto, and S. J. Núnez Jareno, Isotropy theorem for arbitrary-spin cosmological fields, J. Cosmol. Astropart. Phys. 03 (2014) 042.

[44] D. S. Gorbunov and V. A. Rubakov, Introduction to the theory of the early universe: Cosmological perturbations and inflationary theory, arXiv:10.1142/7874.

[45] J. A. R. Cembranos, A. L. Maroto, and S. J. Núnez Jareno, Perturbations of ultralight vector field dark matter, J. High Energy Phys. 02 (2017) 064.

[46] T. S. Pereira, C. Pitrou, and J.-P. Uzan, Theory of cosmological perturbations in an anisotropic universe, J. Cosmol. Astropart. Phys. 09 (2007) 006.

[47] P. A. R. Ade et al. (PlANCK collaboration), Planck 2015 results. XIX. Constraints on primordial magnetic fields, Astron. Astrophys. 594, A19 (2016) A19.

[48] A. Kandus, K. E. Kunze, and C. G. Tsagas, Primordial magnetogenesis, Phys. Rep. 505, 1 (2011).

[49] R. Durrer and A. Neronov, Cosmological magnetic fields: Their generation, evolution and observation, Astron. Astrophys. Rev. 21, 62 (2013).

[50] K. Subramanian, The origin, evolution and signatures of primordial magnetic fields, Rept. Prog. Phys. 79 (2016) 076901.

[51] Y. Yamashita, A. De Felice, and T. Tanaka, Appearance of Boulware-Deser ghost in bigravity with doubly coupled matter, Int. J. Mod. Phys. D 23, 1443003 (2014).

[52] C. de Rham, L. Heisenberg, and R. H. Ribeiro, Ghosts and matter couplings in massive gravity, bigravity and multigravity, Phys. Rev. D 90, 124042 (2014).

[53] C. de Rham, L. Heisenberg, and R. H. Ribeiro, On couplings to matter in massive (bi-)gravity, Classical Quantum Graivty. 32, 035022 (2015).

[54] L. Heisenberg, Quantum corrections in massive bigravity and new effective composite metrics, Classical Quantum Gravity 32, 105011 (2015).

[55] K. Hinterbichler and R. A. Rosen, Note on ghost-free matter couplings in massive gravity and multigravity, Phys. Rev. D 92, 024030 (2015).

[56] L. Heisenberg, More on effective composite metrics, Phys. Rev. D 92, 023525 (2015).

[57] C. de Rham and A.J. Tolley, Vielbein to the rescue? Breaking the symmetric vielbein condition in massive gravity and multigravity, Phys. Rev. D 92, 024024 (2015).

[58] Q.-G. Huang, R. H. Ribeiro, Y.-H. Xing, K.-C. Zhang, and S.-Y. Zhou, On the uniqueness of the non-minimal matter coupling in massive gravity and bigravity, Phys. Lett. B 748, 356 (2015).

[59] A. De Felice, A. E. Gümrükçüoğlu, L. Heisenberg, and S. Mukohyama, Matter coupling in partially constrained vielbein formulation of massive gravity, J. Cosmol. Astropart. Phys. 01 (2016) 003.

[60] M.S. Turner, Coherent scalar field oscillations in an expanding universe, Phys. Rev. D 28, 1243 (1983).

[61] W. H. Press, B.S. Ryden, and D. N. Spergel, Single Mechanism for Generating Large Scale Structure and Providing Dark Missing Matter, Phys. Rev. Lett. 64, 1084 (1990).

[62] S.-J. Sin, Late time cosmological phase transition and galactic halo as Bose liquid, Phys. Rev. D 50, 3650 (1994).
[63] S. U. Ji and S. J. Sin, Late time phase transition and the galactic halo as a bose liquid: 2 . The Effect of visible matter, Phys. Rev. D 50, 3655 (1994).

[64] J.-w. Lee and I.-g. Koh, Galactic halos as boson stars, Phys. Rev. D 53, 2236 (1996).

[65] W. Hu, R. Barkana, and A. Gruzinov, Cold and Fuzzy Dark Matter, Phys. Rev. Lett. 85, 1158 (2000).

[66] J. Goodman, Repulsive dark matter, New Astron. 5, 103 (2000).

[67] P. J. E. Peebles, Fluid dark matter, Astrophys. J. 534 (2000).

[68] L. Amendola and R. Barbieri, Dark matter from an ultralight pseudo-Goldsone-boson, Phys. Lett. B 642, 192 (2006).

[69] C. G. Boehmer and T. Harko, Can dark matter be a BoseEinstein condensate?, J. Cosmol. Astropart. Phys. 06 (2007) 025.

[70] J.-W. Lee and S. Lim, Minimum mass of galaxies from BEC or scalar field dark matter, J. Cosmol. Astropart. Phys. 01 (2010) 007.

[71] P. Sikivie and Q. Yang, Bose-Einstein Condensation of Dark Matter Axions, Phys. Rev. Lett. 103, 111301 (2009).

[72] P.-H. Chavanis, Growth of perturbations in an expanding universe with Bose-Einstein condensate dark matter, Astron. Astrophys. 537, A127 (2012).

[73] H.-Y. Schive, T. Chiueh, and T. Broadhurst, Cosmic structure as the quantum interference of a coherent dark wave, Nat. Phys. 10, 496 (2014).

[74] L. Hui, J. P. Ostriker, S. Tremaine, and E. Witten, Ultralight scalars as cosmological dark matter, Phys. Rev. D 95, 043541 (2017).

[75] A. Higuchi, Forbidden mass range for spin-2 field theory in de Sitter space-time, Nucl. Phys. B282, 397 (1987).

[76] A. Higuchi, Massive symmetric tensor field in space-times with a positive cosmological constant, Nucl. Phys. B325, 745 (1989).

[77] L. Grisa and L. Sorbo, Pauli-Fierz gravitons on FriedmannRobertson-Walker background, Phys. Lett. B 686, 273 (2010).

[78] M. Fasiello and A. J. Tolley, Cosmological perturbations in massive gravity and the Higuchi bound, J. Cosmol. Astropart. Phys. 11 (2012) 035.

[79] M. Fasiello and A. J. Tolley, Cosmological stability bound in massive gravity and bigravity, J. Cosmol. Astropart. Phys. 12 (2013) 002.

[80] D. Comelli, M. Crisostomi, and L. Pilo, Perturbations in massive gravity cosmology, J. High Energy Phys. 06 (2012) 085.

[81] D. Comelli, M. Crisostomi, and L. Pilo, FRW cosmological perturbations in massive bigravity, Phys. Rev. D 90, 084003 (2014).

[82] A. De Felice, A. E. Gümrükçüoğlu, S. Mukohyama, N. Tanahashi, and T. Tanaka, Viable cosmology in bimetric theory, J. Cosmol. Astropart. Phys. 06 (2014) 037.

[83] K. Aoki, K.-i. Maeda, and R. Namba, Stability of the early universe in bigravity theory, Phys. Rev. D 92, 044054 (2015).

[84] E. Mortsell and J. Enander, Scalar instabilities in bimetric gravity: The Vainshtein mechanism and structure formation, J. Cosmol. Astropart. Phys. 10 (2015) 044.

[85] K. Aoki and S. Mizuno, Vainshtein mechanism in massive gravity nonlinear sigma models, Phys. Rev. D 94, 064054 (2016). 